ESAIM: PROCEEDINGS AND SURVEYS, February 2019, Vol. 65, p. 401-424

B. Bouchard, J.-F. Chassagneux, F. Delarue, E. Gobet and J. Lelong, Editors

\title{
STATISTICAL AND PROBABILISTIC MODELING OF A CLOUD OF PARTICLES COUPLED WITH A TURBULENT FLUID
}

\author{
Ludovic Goudenège ${ }^{1}$, Adam Larat ${ }^{1,6,2}$, Julie Llobell ${ }^{3}$, Marc Massot $^{4}$, \\ David Mercier ${ }^{6}$, Olivier Thomine ${ }^{5}$ And Aymeric Vié ${ }^{1,6}$
}

\begin{abstract}
This paper exposes a novel exploratory formalism, the end goal of which is the numerical simulation of the dynamics of a cloud of particles weakly or strongly coupled with a turbulent fluid. Given the large panel of expertise of the list of authors, the content of this paper scans a wide range of connex notions, from the physics of turbulence to the rigorous definition of stochastic processes. Our approach is to develop reduced-order models for the dynamics of both carrying and carried phases which remain consistant within this formalism, and to set up a numerical process to validate these models. The novelties of this paper lie in the gathering of a large panel of mathematical and physical definitions and results within a common framework and an agreed vocabulary (sections 1 and 2), and in some preliminary results and achievements within this context, section 3 . While the first three sections have been simplified to the context of a gas field providing that the disperse phase only retrieves energy through drag, the fourth section opens this study to the more complex situation when the disperse phase interacts with the continuous phase as well, in an energy conservative manner. This will allow us to expose the perspectives of the project and to conclude.
\end{abstract}

\section{Contents}

Introduction

1. Statistical description of the dynamics of a population of particles 403

1.1. Microscopic scale 404

1.2. Mesoscopic scale 404

2. A population of particles in a turbulent fluid 407

2.1. Classical theories for macroscopic equations for the fluid 407

2.2. Large-scale reduced-order models 409

2.3. Particles in turbulence 411

3. Consistency of modeling approaches with numerical cases 412

3.1. Synthetic turbulence 412

3.2. Simplified one-dimensional case 413

1 Fédération de Mathématiques de CentraleSupélec, CNRS FR-3487, CentraleSupélec, Université Paris-Saclay, 9 rue Joliot Curie, 91190 Gif-sur-Yvette cedex, France

2 Univ. Grenoble Alpes, CNRS, Grenoble INP, LJK, 38000 Grenoble, France

3 Université Côte d'Azur, Inria, CNRS, LJAD, Parc Valrose, F-06108 Nice, France

${ }^{4}$ CMAP, École Polytechnique, Route de Saclay, 91128 Palaiseau cedex, France

5 CEA/DEN/DANS, 91191 Gif-sur-Yvette cedex, France

${ }^{6}$ Laboratoire EM2C, CNRS, CentraleSupélec, Université Paris-Saclay, 3 rue Joliot Curie, 91192 Gif-sur-Yvette cedex, France

(C) EDP Sciences, SMAI 2019

This is an Open Access article distributed under the terms of the Creative Commons Attribution License (http://creativecommons.org/licenses/by/4.0), which permits unrestricted use, distribution, and reproduction in any medium, provided the original work is properly cited. 
3.3. Higher dimensionality 417

4. Towards two-way coupled systems 417

4.1. Example of the Burgers equation 418

4.2. Particle-laden case with Lagrangian particles 419

4.3. Eulerian modeling 419

5. Conclusions 422

References 422

\section{INTRODUCTION}

Many applications involve the transport of a disperse phase (particles, droplets, bubbles) coupled with a turbulent fluid: spray combustion, fluidized beds, soot dynamics... In the standard case, the evolution of the carrier phase can be described by a deterministic system of equations such as the averaged Navier-Stokes equations (RANS models). However, in the strongly coupled case the evolution equations are unclosed due to the exchange term with the particles $[10,13$. Often, models proposed in the literature only consider the influence of the carrier fluid on the disperse phase and neglect its retroactive consequences, or, at best, limit it to a global balance between the two phases [23. In particular, these simplifying hypotheses allow to decouple the inaccuracies coming from the approximate resolution of each phase.

But, one of the main difficulties in the derivation of a consistent model for the strongly coupled evolution of a cloud of particles within a turbulent flow, is that inaccuracies arise both from the chaotic behavior of the fluid $9,28,42$, and from the initial properties of the particles, such as their starting positions and velocities. Therefore, the proper level of "modeling" consists in making consistant assumptions about the properties of the stochastic processes involved in the global dynamics of both phases. Even if some advances have been made in the field [15], the problematic is far from being closed.

In order to better understand the coupling of the inaccuracies coming from both phases, we split the construction of the fluid dynamic model into four main steps, corresponding to four levels of modeling. We express the links between these levels both for the carrier fluid and for the disperse phase, in order to better understand the influence of the small scales on the highest level of modeling. The link between the various levels of modeling can be fully justified based on asymptotic behavior or rely on some assumptions, which have to be detailed. Besides, each of the passing to the limit between the levels does not occur at the same scale for the two phases. Although the carrier fluid is constituted of molecules, while the dispersed particles seldom reach a micrometrical size, the description of each phase can start at the microscopic level (or molecular level). From there, one can derive larger-scale models based on rigorous asymptotics or on valid assumptions, by first looking at an intermediate mesoscopic scale, with a statistical description rather than a description of each particles in a deterministic way (e.g. the Boltzmann equation), and then consider close-to-equilibrium regimes that we will call the macroscopic or fluid scale (e.g. Euler or Navier-Stokes equations). These four different levels of modeling are sketched level-by-level in the following items list:

- Microscopic: at the scale of atomes, molecules for the carrier gas or particules of the disperse phase. Generally speaking, one may say "at the scale of the indivisible". The medium is here modeled by a very large number of ODEs.

- Macroscopic: at the scale of the continuum. Fluids (liquid, gaz, spray,...) are now seen as a continuous medium. It is modeled by a system of PDEs derived either from kinetic theory [6, 12] or relying on rational thermodynamics 34,39 .

- Mesoscopic: the transition from the micro to the macro scale can rely on an intermediate scale, called "mesoscopic", at which the medium is modeled a statistical manner. At this level, the fluid is modeled by the transport equation of a number density function (NDF) of particles.

- Reduced-Order: despite all the complexity reduction already performed, the simulation of all the scales (Direct Numerical Simulation - DNS - of either a fluid-fluid model or a fluid kinetic model...) is 
far from being reachable. An additional order reduction is usually performed by splitting the solution into a significant part $\bar{\phi}$ and a residual $\phi^{\prime}: \phi=\bar{\phi}+\phi^{\prime}$. In general, the residual is removed and its action on the resolved part is modeled by a chosen underlying random process.

Throughout this paper the term significant part is kept general on purpose: it could denote one of the numerous choice of decomposition of the macroscopic (or mesoscopic) solution we are looking for into a numerically resolved and an unresolved part (see subsection 2.2.2 for more details). To give an insight of historical context, the usual method is traditionally referred to as a Large Eddy Simulation (LES) of the particulate flow, which means that only the features of the flow at a scale greater than a characteristic cut-off size are computed. The smallest scales, called subscales, need to be modeled from the computed variables in both carrying and disperse phases. As proposed by Pope [29], we chose to place ourselves in a probabilistic formalism where the closure in performed by the definition of a probabilistic process for the residuals. This closure can be seen as a probabilistic mapping between the reduction of the non-linear terms of the solved macroscopic PDEs and the resolved variables (see subsection 2.2.2). As a consequence, defining what is called a "subgrid scale (SGS)" model is equivalent to making a choice for this mapping. This is what we are looking for in this project.

An ideal model for the numerical simulation of a turbulent flow loaded with dispersed particles would be a global reduced-order model for both phases, where the residual part would take into account the strong coupling between both phases (mass, momentum and energy are exchanged in a bidirectional manner and globally conserved). We think that the formalism introduced in [29], and rapidly sketched in the previous paragraph, is a good starting point. We also believe that the stochastic model of the unresolved fluctuations has a root at the smallest level of description for both phases, where a rigorous asymptotic derivation is valid and where we then start our exploratory study by considering an idealistic modeling with additional stochastic processes on both phases, and then try to derive a global large-scale reduced-order model for the dynamics of the strongly coupled system, which remains reliable, accurate and consistant with the underlying description of the physical system.

This paper is divided into four sections. In a Section 1, we give a statistical description at micro and mesoscale which are the beginning of all macroscopic descriptions, with a theorem in the infinite population limit. It explains the link between a system of a large number of ODEs at the microscopic level and a PDE on a Probability Density Function (PDF) of existence of the particles. In Section 2 we describe in a very condensed manner the other levels of continuous description, while staying as consistant as possible. This leads us to a very general definition of turbulence and to the probabilistic framework for the modeling of the subscales in the context described by Pope [29]. In particular we explain the derivation of a reduced-order model for the disperse phase only, when the underlying carrying continuous gas field is supposed to be perfectly known and is not perturbed by the presence of the particles. Section 3 presents a numerical process intended to validate the reduced-order models possibly created within this micro/micro to reduced-order context, by looking at the statistics missed by the disperse field when the underlying gas velocity field has been reduced (for example filtered). In particular, we show that it seems hard to build a reduced-order turbulent model for the dynamics of a 1D spray, but that the situation improves with higher dimensionality. Finally, Section 4 opens the discussion on the construction of a consistent model for two-way coupled systems. This section being preliminary, this will allow us to expose the perspectives of the current project and to conclude the paper.

\section{Statistical Description of The Dynamics of a POPUlation of PARTiCLES}

This section describes the dynamics of a population of particles from micro to meso-scale. This is the beginning of all work implying complex dynamics of turbulent particules-laden flows. This gathering represents a real team effort, especially in finding a common vocabulary between those of us more physics-oriented and those more used to the theory of probability and of stochastic processes. As already said in the previous paragraph, what is written here is valid for both carrying and disperse phases, only the passages to the limit do not occur at the same scales. 


\subsection{Microscopic scale}

The studied domain $\mathbb{X} \subset \mathbb{R}^{3}$ is filled with a cloud of $N$ identical spherical particles, moving into void or supported by a carrying gas. Assuming that the three degrees of freedom in rotation of each particle can be ignored, the dynamics of the system is described by the $6 N$ parameters (velocity are in $\mathbb{R}_{C}^{3}:=\mathbb{R}^{3}$ ):

$$
\boldsymbol{Z}(t)=\left(\boldsymbol{X}_{1}(t), \boldsymbol{C}_{1}(t), \ldots, \boldsymbol{X}_{N}(t), \boldsymbol{C}_{N}(t)\right) \in \boldsymbol{\zeta}^{N}:=\left(\mathcal{X} \times \mathbb{R}_{C}^{3}\right)^{N}
$$

or equally by the empirical measure or normalized counting measure: $\mu_{t}^{N}[\boldsymbol{Z}]=\frac{1}{N} \sum_{i=1}^{N} \delta_{\boldsymbol{X}_{i}(t)} \delta_{\boldsymbol{C}_{i}(t)}$.

If the particles are immersed within an external field $\boldsymbol{G}(t, \boldsymbol{X}, \boldsymbol{C})$, interact through a collision kernel $\boldsymbol{F}(\boldsymbol{X}, \boldsymbol{C})$ and are possibly subject to independent Brownian motions of intensity $\sigma$, the state variables (1) evolve with the following system of $6 N$ ODEs:

$$
\left\{\begin{array}{rl}
d \boldsymbol{X}_{i} & =\boldsymbol{C}_{i}(t) d t \\
d \boldsymbol{C}_{i} & =\boldsymbol{G}\left(t, \boldsymbol{X}_{i}, \boldsymbol{C}_{i}\right) d t+\boldsymbol{F} * \mu_{t}^{N}\left(\boldsymbol{X}_{i}, \boldsymbol{C}_{i}\right) d t+\sqrt{2} \sigma d \boldsymbol{W}_{i}(t),
\end{array} \quad i=1, \ldots, N .\right.
$$

Then, given an initial condition $\boldsymbol{Z}_{0}=\left(\boldsymbol{X}_{1}^{0}, \boldsymbol{C}_{1}^{0}, \ldots, \boldsymbol{X}_{N}^{0}, \boldsymbol{C}_{N}^{0}\right)$, which may be deterministic or stochastic, the empirical measure can be indexed by $\boldsymbol{Z}_{0}: \mu_{t}^{N}\left[\boldsymbol{Z}_{0}\right]=\frac{1}{N} \sum_{i=1}^{N} \delta_{\boldsymbol{X}_{i}\left(t, \boldsymbol{Z}_{0}\right)} \delta_{\boldsymbol{C}_{i}\left(t, \boldsymbol{Z}_{0}\right)}$, so that if $V_{\boldsymbol{X}} \times V_{\boldsymbol{C}} \subset \boldsymbol{\zeta}$ is a subset of phase space,

$$
n_{V_{\boldsymbol{X}} \times V_{\boldsymbol{C}}}:=N . \mu_{t}^{N}\left[\boldsymbol{Z}_{0}\right]\left(V_{\boldsymbol{X}} \times V_{\boldsymbol{C}}\right)=\sum_{i=1}^{N} \mathbb{1}_{V_{\boldsymbol{X}}}\left(\boldsymbol{X}_{i}\left(t, \boldsymbol{Z}_{0}\right)\right) \mathbb{1}_{V_{\boldsymbol{C}}}\left(\boldsymbol{C}_{i}\left(t, \boldsymbol{Z}_{0}\right)\right)
$$

is the number of particles from the configuration $\boldsymbol{Z}_{0}$ at time $t=0$, situated within $V_{\boldsymbol{X}}$ and with a velocity belonging to $V_{\boldsymbol{C}}$ at time $t$.

\subsection{Mesoscopic scale}

From now on, the configuration of each particle is denoted by $\boldsymbol{z}_{i}=\left(\boldsymbol{X}_{i}, \boldsymbol{C}_{i}\right) \in \mathbb{X} \times \mathbb{R}_{\boldsymbol{C}}^{3}$, for all $i=1, \ldots, N$. The collision kernel $\boldsymbol{F}$ simulates the interaction between the particles and it thus seems fair to have $\boldsymbol{F}(-\boldsymbol{z})=-\boldsymbol{F}(\boldsymbol{z})$, which implies $\boldsymbol{F}(\mathbf{0})=\mathbf{0}$.

Let us consider that the particles are exchangeable at initial time, which means that their initial distribution $\mu_{0}^{N} \in \mathbb{R}^{2 d}$ is invariant by permutation of the $N$ variables. This invariance therefore remains satisfied at any time $t>0$ and in particular, the $N$ particles must follow the same one-particle law in $\mathbb{R}^{2 d}$, denoted $\mu_{t}^{(1)}$, which is what we are looking for in this subsection. First, if $A$ is a Borelian in $\mathbb{R}^{2 d}$,

$$
\mathbb{E}\left[\mu_{t}^{N}(A)\right]=\mathbb{E}\left[\frac{1}{N} \sum_{i=1}^{N} \delta_{\boldsymbol{z}_{i}(t)}(A)\right]=\frac{1}{N} \sum_{i=1}^{N} \mathbb{P}\left[\boldsymbol{z}_{i}(t) \in A\right]=\mathbb{P}\left[\boldsymbol{z}_{1}(t) \in A\right]=\mu_{t}^{(1)}(A) .
$$

Then, we recall $\boldsymbol{Z}(t)=\left(\boldsymbol{z}_{1}(t), \ldots, \boldsymbol{z}_{N}(t)\right)$, later simply noted $\boldsymbol{Z}_{t}$, and we introduce the following function

$$
\boldsymbol{H}(t, \boldsymbol{Z})=\left(\boldsymbol{c}_{1}, \boldsymbol{G}\left(t, \boldsymbol{z}_{1}\right)+\frac{1}{N} \sum_{j=1}^{N} \boldsymbol{F}\left(\boldsymbol{z}_{1}-\boldsymbol{z}_{j}\right), \cdots, \boldsymbol{c}_{N}, \boldsymbol{G}\left(t, \boldsymbol{z}_{N}\right)+\frac{1}{N} \sum_{j=1}^{N} \boldsymbol{F}\left(\boldsymbol{z}_{N}-\boldsymbol{z}_{j}\right)\right),
$$

and the $2 d \times N$ diagonal matrix, denoted $\Sigma$, with zero ( $d$ times) and $\sigma$ ( $d$ times), repeated $N$ times along the diagonal. Thus, equation (2) can be rewritten

$$
d \boldsymbol{Z}(t)=\boldsymbol{H}(t, \boldsymbol{Z}(t)) d t+\sqrt{2} \Sigma d \boldsymbol{W}(t) .
$$


Next, if $T \in] 0,+\infty[$ is an arbitrary final time, then for any function $\Phi:(t, \boldsymbol{x}) \longmapsto \Phi(t, \boldsymbol{x})$ such that

$$
t \longmapsto \Phi(t, .) \in \mathcal{C}^{1}(0, T) \text { and } x \longmapsto \Phi(., x) \in \mathcal{C}_{c}^{\infty}\left(\mathbb{R}^{2 d}\right)
$$

Itô's formula gives us:

$$
\begin{aligned}
\Phi\left(T, \boldsymbol{Z}_{T}\right)-\Phi\left(0, \boldsymbol{Z}_{0}\right) & =\sigma^{2} \sum_{i=1}^{N} \int_{0}^{T} \Delta_{\boldsymbol{c}_{i}} \Phi\left(t, \boldsymbol{Z}_{t}\right) d t+\int_{0}^{T} \frac{\partial}{\partial t} \Phi\left(t, \boldsymbol{Z}_{t}\right) d t \\
& +\sum_{i=1}^{N} \int_{0}^{T} \nabla_{\boldsymbol{x}_{i}} \Phi\left(t, \boldsymbol{Z}_{t}\right) \cdot d \boldsymbol{Z}_{t}+\sum_{i=1}^{N} \int_{0}^{T} \nabla_{\boldsymbol{c}_{i}} \Phi\left(t, \boldsymbol{Z}_{t}\right) \cdot d \boldsymbol{Z}_{t} \\
& =\sigma^{2} \sum_{i=1}^{N} \int_{0}^{T} \Delta_{\boldsymbol{c}_{i}} \Phi\left(t, \boldsymbol{Z}_{t}\right) d t+\int_{0}^{T} \frac{\partial}{\partial t} \Phi\left(t, \boldsymbol{Z}_{t}\right) d t \\
& +\sum_{i=1}^{N} \int_{0}^{T} \nabla_{\boldsymbol{x}_{i}} \Phi\left(t, \boldsymbol{Z}_{t}\right) \cdot \boldsymbol{H}\left(t, \boldsymbol{Z}_{t}\right) d t+\sqrt{2} \sum_{i=1}^{N} \int_{0}^{T} \nabla_{\boldsymbol{x}_{i}} \Phi\left(t, \boldsymbol{Z}_{t}\right) \cdot \Sigma d \boldsymbol{W}_{t} \\
& +\sum_{i=1}^{N} \int_{0}^{T} \nabla_{\boldsymbol{c}_{i}} \Phi\left(t, \boldsymbol{Z}_{t}\right) \cdot \boldsymbol{H}\left(t, \boldsymbol{Z}_{t}\right) d t+\sqrt{2} \sum_{i=1}^{N} \int_{0}^{T} \nabla_{\boldsymbol{c}_{i}} \Phi\left(t, \boldsymbol{Z}_{t}\right) \cdot \Sigma d \boldsymbol{W}_{t},
\end{aligned}
$$

where $\left[\nabla_{\boldsymbol{x}_{i}} \Phi\left(t, \boldsymbol{Z}_{t}\right) \cdot\right]$ and $\left[\nabla_{\boldsymbol{c}_{i}} \Phi\left(t, \boldsymbol{Z}_{t}\right) \cdot\right]$ denote projection operators on the respective lines of $\boldsymbol{x}_{i}$ and $\boldsymbol{c}_{i}$. Taking the expectation we get:

$$
\begin{aligned}
\mathbb{E}\left[\Phi\left(T, \boldsymbol{Z}_{T}\right)-\Phi\left(0, \boldsymbol{Z}_{0}\right)\right] & =\sigma^{2} \sum_{i=1}^{N} \int_{0}^{T} \mathbb{E}\left[\Delta_{\boldsymbol{c}_{i}} \Phi\left(t, \boldsymbol{Z}_{t}\right)\right] d t+\int_{0}^{T} \mathbb{E}\left[\frac{\partial}{\partial t} \Phi\left(t, \boldsymbol{Z}_{t}\right)\right] d t \\
& +\sum_{i=1}^{N} \int_{0}^{T} \mathbb{E}\left[\nabla_{\boldsymbol{x}_{i}} \Phi\left(t, \boldsymbol{Z}_{t}\right) \cdot \boldsymbol{H}\left(t, \boldsymbol{Z}_{t}\right)\right] d t+\sum_{i=1}^{N} \int_{0}^{T} \mathbb{E}\left[\nabla_{\boldsymbol{c}_{i}} \Phi\left(t, \boldsymbol{Z}_{t}\right) \cdot \boldsymbol{H}\left(t, \boldsymbol{Z}_{t}\right)\right] d t
\end{aligned}
$$

Next, for any $\nu \in \mathcal{C}^{0}\left([0, T], \mathcal{M}\left(\mathbb{R}^{2 d}\right)\right)$ and for any $t \in[0, T]$, we define the measure of $\mathbb{R}^{2 d}: \nu_{t}(\cdot)=\nu(t, \cdot)$. Then, for any $\Phi$ such as in $(3)$, we introduce the following linear form on the space $\mathcal{C}^{0}\left([0, T], \mathcal{M}\left(\mathbb{R}^{2 d}\right)\right)$ :

$$
\langle\nu, \Phi\rangle_{T}=\int_{0}^{T} \int_{\mathbb{R}^{2 d}} \Phi(t, \boldsymbol{z}) d \nu_{t}(\boldsymbol{z}) d t
$$

By abuse of notation, this linear form in space and time will also be denoted by $\left\langle\nu_{t}, \Phi\right\rangle_{T}$. In the specific case when $\nu_{t}=\mu_{t}^{(N)}$, we look at the $N$-joint law followed by the $N$ particules. Since this is the law followed by $\boldsymbol{Z}_{t}$, for any $\Phi$ defined by $(3)$, the linear form also writes $\left\langle\nu_{t}, \Phi\right\rangle_{T}=\int_{0}^{T} \mathbb{E}\left[\Phi\left(t, Z_{t}\right)\right] d t:=\left\langle\mu_{t}^{(N)}, \Phi\right\rangle_{T}$. Using this dual formulation, we can now extend the definition of the partial derivatives to the measures of $\mathbb{R}^{2 d}$, and we have:

$$
\begin{aligned}
& \int_{0}^{T} \mathbb{E}\left[\nabla_{\boldsymbol{x}_{i}} \Phi\left(t, \boldsymbol{Z}_{t}\right) \cdot \boldsymbol{H}\left(t, \boldsymbol{Z}_{t}\right)\right] d t:=-\left\langle\boldsymbol{c}_{i} \cdot \nabla_{\boldsymbol{x}_{i}} \mu_{t}^{(N)}, \Phi\right\rangle_{T} \\
& \int_{0}^{T} \mathbb{E}\left[\nabla_{\boldsymbol{c}_{i}} \Phi\left(t, \boldsymbol{Z}_{t}\right) \cdot \boldsymbol{H}\left(t, \boldsymbol{Z}_{t}\right)\right] d t:=-\left\langle\nabla_{\boldsymbol{c}_{i}} \cdot\left[\left(\boldsymbol{G}\left(., \boldsymbol{z}_{i}\right)+\frac{1}{N} \sum_{j=1}^{N} \boldsymbol{F}\left(\boldsymbol{z}_{i}-\boldsymbol{z}_{j}\right)\right) \mu_{t}^{(N)}\right], \Phi\right\rangle_{T}, \\
& \int_{0}^{T} \mathbb{E}\left[\Delta_{\boldsymbol{c}_{i}} \Phi\left(t, \boldsymbol{Z}_{t}\right)\right] d t:=\left\langle\Delta_{\boldsymbol{c}_{i}} \mu_{t}^{(N)}, \Phi\right\rangle_{T} .
\end{aligned}
$$


Since $\Phi$ does not have a compact support in time, integration by part requires to keep the boundary terms, and the time partial derivative of $\mu_{t}^{(N)}$ defines as:

$$
\int_{0}^{T} \mathbb{E}\left[\frac{\partial}{\partial t} \Phi\left(t, \boldsymbol{Z}_{t}\right)\right] d t-\mathbb{E}\left[\Phi\left(T, \boldsymbol{Z}_{T}\right)-\Phi\left(0, \boldsymbol{Z}_{0}\right)\right]:=-\left\langle\partial_{t} \mu_{t}^{(N)}, \Phi\right\rangle_{T} .
$$

To sum up, thanks to the Itô's formula, we have obtained a weak form of the equation followed by the law $\mu_{t}^{(N)}$ of $\boldsymbol{Z}(t)$, which is of the Fokker-Planck type:

$$
\left\langle\partial_{t} \mu_{t}^{(N)}+\sum_{i=1}^{N} \boldsymbol{c}_{i} \cdot \nabla_{\boldsymbol{x}_{i}} \mu_{t}^{(N)}+\sum_{i=1}^{N} \nabla_{\boldsymbol{c}_{i}} \cdot\left(\left[\boldsymbol{G}\left(., \boldsymbol{z}_{i}\right)+\frac{1}{N} \sum_{j=1}^{N} \boldsymbol{F}\left(\boldsymbol{z}_{i}-\boldsymbol{z}_{j}\right)\right] \mu_{t}^{(N)}\right), \Phi\right\rangle_{T}=\sigma^{2}\left\langle\sum_{i=1}^{N} \Delta_{\boldsymbol{c}_{i}} \mu_{t}^{(N)}, \Phi\right\rangle_{T} .
$$

We have now generalized the results given by Bolley in [5] to a time dependent transport term $\boldsymbol{G}$. See also 38 and 40 for more details on the mean-field theory.

However, equation (4) is a weak formulation of a PDE on the $N$-particles joint law, when what we are looking for is the equation ruling the one-particle law $\mu_{t}^{(1)}$, which is the marginal of $\mu_{t}^{(N)}$ for particle 1. By integrating Eq. (4) over all the particles but the first one, we get that, in the weak sense, $\mu_{t}^{(1)}$ follows:

$$
\partial_{t} \mu_{t}^{(1)}+\boldsymbol{c} \cdot \nabla_{\boldsymbol{x}} \mu_{t}^{(1)}+\nabla_{\boldsymbol{c}} \cdot\left(\boldsymbol{G}_{t} \mu_{t}^{(1)}+\int_{\boldsymbol{z}_{2} \in \mathbb{R}^{2 d}} \boldsymbol{F}\left(\boldsymbol{z}-\boldsymbol{z}_{2}\right) \mu_{t}^{(2)}\left(\boldsymbol{z}, \boldsymbol{z}_{2}\right)\right)=\sigma^{2} \Delta_{\boldsymbol{c}} \mu_{t}^{(1)}
$$

In this expression, $\mu_{t}^{(2)}$ is the 2-particles joint probability. In order to close equation (5), we would like to express it as a function of $\mu_{t}^{(1)}$.

To do so, we suppose that the initial data $\boldsymbol{Z}_{0}$ are exchangeable and all follow the same law $f_{0}$ on $\mathbb{R}^{2 d}$. Then, we introduce an intermediate law, as the solution for $t>0$ and $(\boldsymbol{x}, \boldsymbol{c}) \in \mathbb{R}^{2 d}$, of the following equation with initial data $f_{0}$ :

$$
\frac{\partial}{\partial t} f_{t}+\boldsymbol{c} \cdot \nabla_{\boldsymbol{x}} f_{t}+\nabla_{\boldsymbol{c}} \cdot\left[\left(\boldsymbol{G}+\boldsymbol{F} * f_{t}\right) f_{t}\right]=\sigma^{2} \Delta_{\boldsymbol{c}} f_{t}
$$

where $\boldsymbol{F}$ and $\boldsymbol{G}$ are now supposed to be Lipschitz functions with respect to the variable $\boldsymbol{x} \in \mathbb{R}^{2 d}$ and $\boldsymbol{G}$ is continuous in the time variable. Next, for $i \in 1, \ldots, N$, let $\overline{\boldsymbol{z}}_{i}(t)$ be the solution of the following system with initial data $\overline{\boldsymbol{z}}_{i}(0)=\boldsymbol{z}_{i}(0)$ :

$$
\left\{\begin{array}{l}
d \overline{\boldsymbol{x}}_{i}(t)=\overline{\boldsymbol{c}}_{i}(t) d t \\
d \overline{\boldsymbol{c}}_{i}(t)=\boldsymbol{G}\left(t, \overline{\boldsymbol{z}}_{i}(t)\right) d t+\boldsymbol{F} * f_{t}\left(\overline{\boldsymbol{z}}_{i}(t)\right) d t+\sqrt{2} \sigma d \boldsymbol{W}_{i}(t)
\end{array}\right.
$$

Then, the fictive particles $\overline{\boldsymbol{z}}_{i}$ evolve in the field $\boldsymbol{F} * f_{t}$ generated by the distribution $f_{t}$, while the $\boldsymbol{z}_{i}$ particles evolve in the $\boldsymbol{F} * \mu_{t}^{N}$ field, generated by the empirical measure $\mu_{t}^{N}$. Itô's formula gives once more the PDE followed by $\bar{z}_{i}$ in the weak sense, and we now wish to show that this measure converges to $f_{t}$ when the number $N$ of particles tends to infinity.

We denote $|(\boldsymbol{x}, \boldsymbol{c})|=\sqrt{|\boldsymbol{x}|^{2}+|\boldsymbol{c}|^{2}}$ and for $p>1$ we define

$$
\mathbb{P}_{p}\left(\mathbb{R}^{2 d}\right)=\left\{\mu \text { Borelian probabilistic measures on } \mathbb{R}^{2 d} \text { such that } p \text {-momentum } \int_{\mathbb{R}^{2 d}}|(\boldsymbol{x}, \boldsymbol{c})|^{p} d \mu(\boldsymbol{x}, \boldsymbol{c})<\infty\right\} .
$$

The Wasserstein distance of order $p$ between two measures $\mu$ and $\bar{\mu}$ of $\mathbb{P}_{p}\left(\mathbb{R}^{2 d}\right)$ is defined by

$$
W_{p}(\mu, \bar{\mu})=\inf _{\boldsymbol{Z}, \bar{Z}} \sqrt[p]{\mathbb{E}\left[|\boldsymbol{Z}-\overline{\boldsymbol{Z}}|^{p}\right]}
$$


where $\boldsymbol{Z}$ and $\bar{Z}$ are stochastic variables of law $\mu$ and $\bar{\mu}$ respectively. Then, following the lines of 5 ,

Theorem 1.1. we obtain the explicit convergence rates:

1) $W_{2}\left(\mu_{t}^{(1)}, f_{t}\right)^{2} \leqslant \mathbb{E}\left[\left|z_{1}(t)-\bar{z}_{1}(t)\right|^{2}\right] \leqslant \frac{C}{N}$,

2) $W_{2}\left(\mu_{t}^{(k)}, f_{t}^{\otimes k}\right)^{2} \leqslant \mathbb{E}\left[\left|\left(\boldsymbol{z}_{1}(t), \ldots, \boldsymbol{z}_{k}(t)\right)-\left(\overline{\boldsymbol{z}}_{1}(t), \ldots, \overline{\boldsymbol{z}}_{k}(t)\right)\right|^{2}\right] \leqslant \frac{C k}{N}$,

3) Let $\Phi$ be a Lipschitz function in the second variable, then

$$
\mathbb{E}\left[\left|\int_{\mathbb{R}^{2 d}} \Phi \mu_{t}^{N}-\int_{\mathbb{R}^{2 d}} \Phi f_{t}\right|\right] \leqslant \frac{C}{N}\|\Phi\|_{1}^{2}
$$

In other words, this means that:

1) The one-particle law $\mu_{t}^{(1)}$ converges to $f_{t}$ in the Wasserstein distance when $N \rightarrow \infty$,

2) At the limit of an infinite number of particles, the chaos propagates in time; the particles remain uncorrelated during the whole dynamics: $\mu_{t}^{(k)}=f_{t}^{\otimes k}=\underbrace{f_{t} \otimes \cdots \otimes f_{t}}_{\mathrm{k} \text { times }}$. In particular, one recovers the famous molecular chaos assumption of Boltzmann:

$$
\mu_{t}^{(2)}=\mu_{t}^{(1)} \otimes \mu_{t}^{(1)}
$$

3) The weak convergence of the empirical measure $\mu_{t}^{N}$ to $f_{t}$.

Finally, equation (5) is now closed rigorously thanks to the molecular chaos propagation in the context of Lipschitz-regular interactions (external $\boldsymbol{G}$ or between particles $\boldsymbol{F}$ ), 5, 40. However, when the interactions are less regular, which is the case for the Boltzmann equation (9) below, an increasing number of positive results let us think that equation (8) remains correct, [21, 41]. Nonetheless, no rigorous demonstration is nowadays available.

\section{A population of particles in a turbulent Fluid}

In the previous section, a general kinetic equation at meso-scale has been derived for a population of "particles" (molecules, droplets, solid particles). As this point, one can be interested in deriving a two-way coupled system of kinetic equations for the carrying fluid and the particles. However, as mentioned in [7], there is a large difference of scales between the size of the molecules of the carrying fluid and the particles in the spray constituting the cloud of particles and a fully coupled meso-scale for both phases does not provide the good framework in terms of asymptotical behavior in order to properly get the two-way coupled dynamics. Instead, we use the classical strategy of first deriving macroscopic equations for the fluid, and then coupling them to the particle equations, either at micro-scale or at meso-scale, even if we thus introduce a coupling scale since the particles are assumed to be point-particles [43]. In the following, we first present the Euler and Navier-Stokes equations that can describe a carrying fluid, with an emphasis on the underlying assumptions at the kinetic level. In a context where dealing with the whole range of scales of the fluid is not accessible, we detail a general strategy for generating large-scale reduced-order models, and we show how it can be taken into account for the description of the particle dynamics at the microscopic level.

\subsection{Classical theories for macroscopic equations for the fluid}

In the context of gas dynamics and rarefied gas dynamics, in the Boltzmann-Grad limit, one can obtain the classical non-dimensional Botlzmann equation:

$$
\partial_{t} f+\boldsymbol{c} \cdot \partial_{\boldsymbol{x}} f+\partial_{\boldsymbol{c}}\left(\boldsymbol{F}_{\mathrm{ext}} f\right)=\frac{1}{\mathrm{Kn}} Q(f, f),
$$


where $\mathrm{Kn}=\frac{\lambda}{L}$ is the Knudsen number, ratio between the mean free path $\lambda$ and a characteristic size of observation $L$, and where the quadratic collision operator $Q$ writes:

$$
Q(f, f)=\int_{\mathbb{R}_{\boldsymbol{c}^{*}}^{3}} \int_{S_{\boldsymbol{n}}^{2}}\left(f^{\prime} f_{*}^{\prime}-f f_{*}\right)\left|\left(\boldsymbol{c}-\boldsymbol{c}_{*}\right) \cdot \boldsymbol{n}\right| \sigma\left(\left|\boldsymbol{c}-\boldsymbol{c}_{*}\right|, \boldsymbol{n}\right) d \boldsymbol{n} d \boldsymbol{c}_{*}
$$

where $f_{*}, f^{\prime}$ and $f_{*}^{\prime}$ are customary notations for the function $f$ taken respectively at variable $\boldsymbol{c}_{*}, \boldsymbol{c}^{\prime}:=\frac{\boldsymbol{c}+\boldsymbol{c}_{*}}{2}+$ $\frac{\left|\boldsymbol{c}-\boldsymbol{c}_{*}\right|}{2} \boldsymbol{n}$ and $\boldsymbol{c}_{*}^{\prime}:=\frac{\boldsymbol{c}+\boldsymbol{c}_{*}}{2}-\frac{\left|\boldsymbol{c}-\boldsymbol{c}_{*}\right|}{2} \boldsymbol{n}$.

Remark 2.1. When considering a non self-interacting population of particles, its number density function also follows an equation of the (9) type, where for a number of cases the Knudsen number is infinite: $\mathrm{Kn}=+\infty$, also called a Vlasov equation.

\subsubsection{Euler equations}

For any NDF $f$, one can define its microscopic entropy by $h=f \log f$. It can be understood as a local uncertainty rate. Then, the macroscopic entropy reads: $\mathcal{H}(t, \boldsymbol{x})=\int_{\mathbb{R}^{3}} f(t, \boldsymbol{x}, \boldsymbol{c}) \log f(t, \boldsymbol{x}, \boldsymbol{c}) d \boldsymbol{c}$, and one can show that when $f$ is a solution of the Boltzmann equation (9), its macroscopic entropy decreases: $\frac{d \mathcal{H}}{d t} \leq 0$. When the minimum $\mathcal{H}_{\text {min }}$ is reached, $\log f$ must be a collisional invariant and this implies that the velocity distribution $f$ is a Maxwell-Boltzmann distribution [12]:

$$
f_{e q}(\boldsymbol{c})=\exp \left(a_{0}+\boldsymbol{a}_{\mathbf{1}} \cdot \boldsymbol{c}+a_{2} \frac{\left|\boldsymbol{c}^{2}\right|}{2}\right)=\frac{\rho}{(2 \pi \beta)^{2 / 3}} \exp \left(-\frac{|\boldsymbol{c}-\boldsymbol{u}|^{2}}{2 \beta}\right)
$$

uniquely defined by its first three moments $\left(\rho=\int_{\mathbb{R}_{\boldsymbol{c}}^{3}} f_{e q}(\boldsymbol{c}) d \boldsymbol{c}, \rho \boldsymbol{u}=\int_{\mathbb{R}_{\boldsymbol{c}}^{3}} \boldsymbol{c}^{1} f_{e q}(\boldsymbol{c}) d \boldsymbol{c}, \rho E=\int_{\mathbb{R}_{\boldsymbol{c}}^{3}} \boldsymbol{c}^{2} f_{e q}(\boldsymbol{c}) d \boldsymbol{c}\right)$. The mapping $\left(a_{0}, \boldsymbol{a}_{\mathbf{1}}, a_{2}\right) \leftrightarrow(\rho, \boldsymbol{u}, \beta)$ is one-to-one. Next, since at thermodynamic equilibrium $\mathcal{H}=\mathcal{H}_{\text {min }}$ the collision term is null, $Q\left(f_{e q}, f_{e q}\right)=0$, the evolution of the Boltzmann equation (9) is now given by the evolution of its three first moments: the Maxwell transfer equations, which are closed and lead to the Euler equations

$$
\left\{\begin{array}{ccc}
\frac{\partial \rho}{\partial t}+\boldsymbol{\nabla} \cdot(\rho \boldsymbol{u}) & =0 \\
\frac{\partial \rho \boldsymbol{u}}{\partial t}+\boldsymbol{\nabla} \cdot(\rho \boldsymbol{u} \otimes \boldsymbol{u}+p \mathbb{I}) & =0 \\
\frac{\partial \rho E}{\partial t}+\boldsymbol{\nabla} \cdot((\rho E+p) \boldsymbol{u}) & =0
\end{array}\right.
$$

In this context, the pressure $p$ appears in the second order centered moment: $p \mathbb{I}=\int_{\mathbb{R}_{\boldsymbol{c}}^{3}}(\boldsymbol{c}-\boldsymbol{u}) \otimes(\boldsymbol{c}-\boldsymbol{u}) f_{e q}(\boldsymbol{c}) d \boldsymbol{c}$.

\subsubsection{Navier-Stokes-Fourier equations}

In the previous paragraph, the collisional process is considered to be extremely efficient: it instantly leads to the local thermodynamical equilibrium in the limit of zero Knudsen number. In the limit of small but strictly positive Knudsen numbers, we look for near equilibrium regimes $\varepsilon=\mathrm{Kn}, \varepsilon<<1$ relying on an expansion of $f$ in $\varepsilon$. A popular derivation relies on the Chapman-Enskog expansion 12. At first order, $f=f_{0}+\varepsilon f_{1}+\circ(\varepsilon)$, which, at orders $1 / \varepsilon$ and 1 , gives in $(9)$ :

$$
Q\left(f_{0}, f_{0}\right)=0 \quad \Longrightarrow \quad f_{0}=f_{e q} \quad \text { and } \quad \partial_{t} f_{e q}+\boldsymbol{c} \cdot \partial_{\boldsymbol{x}} f_{e q}=Q\left(f_{e q}, f_{1}\right)+Q\left(f_{1}, f_{e q}\right)
$$

The latest equation is an integral equation in $f_{1}$ which might be solved for [12] for a single fluid or a mixture of gases involving internal degrees of freedom and lead to the Navier-Stokes equations [6]: 


$$
\left\{\begin{array} { c c } 
{ \frac { \partial \rho } { \partial t } + \quad \boldsymbol { \nabla } \cdot ( \rho \boldsymbol { u } ) } & { = 0 , } \\
{ \frac { \partial \rho \boldsymbol { u } } { \partial t } + \boldsymbol { \nabla } \cdot ( \rho \boldsymbol { u } \otimes \boldsymbol { u } + \mathbb { P } ) } & { = 0 , } \\
{ \frac { \partial \rho E } { \partial t } + \boldsymbol { \nabla } \cdot ( \rho E \boldsymbol { u } + \mathbb { P } \cdot \boldsymbol { u } + \boldsymbol { q } ) } & { = 0 . }
\end{array} \quad \text { where } \left\{\begin{array}{l}
\mathbb{P}=p \mathbb{I}-\frac{\mu}{2}\left(\boldsymbol{\nabla} \boldsymbol{u}+\boldsymbol{\nabla} \boldsymbol{u}^{t}-\frac{2}{3} \boldsymbol{\nabla} \cdot \boldsymbol{u} \mathbb{I}\right) \\
\boldsymbol{q}=-\lambda \boldsymbol{\nabla} T,
\end{array}\right.\right.
$$

and $\mu$ and $\lambda$ are respectively the viscosity and the thermal conductivity.

\subsection{Large-scale reduced-order models}

\subsubsection{Properties of turbulence}

Turbulence is a particular type of flows which can not be rigorously defined. The easiest way to define it is by using the metric of the Reynolds number: $\operatorname{Re}=\frac{u_{f, 0} L_{0}}{\nu}$, where $u_{f, 0}$ is a characteristic speed of the fluid, $L_{0}$ is a characteristic length scale of the system and $\nu=\frac{\mu}{\rho}$ is the kinematic viscosity of the fluid. We will say that a fluid exhibits a turbulent behavior, when its Reynolds number is high. The limit Reynolds number depends on the considered experiment and on the operating condition. However, the flow is generally turbulent when $\operatorname{Re}>>10^{3}$.

Turbulent flows share in common their chaotic behavior. For deterministic systems, there are multiple definitions of chaos, but in this context we choose to say that turbulent flows all are :

- highly sensitive to the initial conditions of the system. The present determines the future, but the approximate present does not approximately describe the future. For instance, we say that $x_{0}$ is a highly sensitive initial conditions, if for all $L_{0}>M>0$ and for all $\delta>0$, there exists another close initial data $y_{0}$ and an arbitrary time $t>0$ such that

$$
\left|x_{0}-y_{0}\right|<\delta \text { and }\left|x\left(t ; x_{0}\right)-y\left(t ; y_{0}\right)\right| \geq M .
$$

- topologically transitive, in the sense that for every pair of non-empty open sets $U \subset X$ and $V \subset X$, there is an arbitrary time $t>0$ such that

$$
\left\{x\left(t ; x_{0}\right) \in X: x_{0} \in U\right\} \cap V \neq \emptyset .
$$

From an experimental point of view, some observations have been made on turbulent fluid flows. The main ones are expressed by Kolmogorov [28, p.190].

- At sufficiently high Reynolds number, the small-scale turbulent motions are statistically isotropic. They follow a universal form that is uniquely determined by the viscosity $\nu$ and the energy dissipation $\varepsilon$.

- The viscosity also defines a cut-off size $\eta_{K}$, called the Kolmogorov scale, below which all the inertia of the flow is dissipated.

- Between the characteristic length $L_{0}$ and $\eta_{K}$, there is an intermediate range of scales, called the inertial range, where the statistics of motion have a universal form that is uniquely determined by the dissipation $\varepsilon$ and is independent of the viscosity $\nu$. Through dimensional analysis, we get that within this range, the turbulent kinetic energy decreases as: $E(|\boldsymbol{k}|) \propto|\boldsymbol{k}|^{-\frac{5}{3}}$, with $\boldsymbol{k}$ the wavenumber.

\subsubsection{Reduced description of turbulence}

It is commonly admitted that the macroscopic Navier-Stokes equations contain the turbulence defined above, in the sense that these equations present solutions which have all the properties listed in paragraph 2.2.1. Nonetheless, in practice the domain size, denoted by $|\mathbb{K}|$, and the dissipative cut-off scale $\eta_{K}$, may be separated 
by many orders of magnitude. In this context, the Direct Numerical Simulation of the Navier-Stokes equations is rapidly unreachable, since the number of needed computational cells will be at least of the order of $\left(\eta_{K} /|\mathbb{X}|\right)^{3}$, not speaking about the potential high number of degrees of freedom per cell.

Therefore, while staying very generic, we consider a decomposition of the solution into a significant part and a residual: if $\phi$ is a quantity of interest, we consider its reduction $\bar{\phi}$ on the space of significant data and thus write $\phi=\bar{\phi}+\phi^{\prime}$. This significant part could be an ensemble average, a filtering, a spatial or a temporal average or even a modal decomposition. The goal is always to reduce the size of the information needed to entirely represent the chosen significant part, hence the name reduced-order model.

Now, the reduction operator - is applied directly on the macroscopic equations Eq. 12 - 13 . For example, when considering the incompressible version of the Navier-Stokes equation, assuming commutativity between all implied linear operators, one gets:

$$
\begin{aligned}
\nabla \cdot \overline{\boldsymbol{u}_{f}} & =0, \\
\partial_{t}\left(\overline{\boldsymbol{u}_{f}}\right)+\overline{\left(\boldsymbol{u}_{f} \cdot \nabla\right) \boldsymbol{u}_{f}}-\nu \nabla^{2} \overline{\boldsymbol{u}_{f}} & =-\frac{1}{\rho_{f}} \nabla \bar{p},
\end{aligned}
$$

with $\boldsymbol{u}_{f}$ the fluid velocity, $\rho_{f}$ its density (constant for incompressible fluids), $\nu$ its kinematic viscosity, $p$ the pressure field and $\nabla^{2}$ is a Laplacian operator.

The main difficulty now lies in the reduction of the non-linear terms. Indeed, nothing indicates that there exists an application giving $\overline{\left(\boldsymbol{u}_{f} \cdot \nabla\right) \boldsymbol{u}_{f}}$ as a function of $\overline{\boldsymbol{u}_{f}}$. Thus, Eq. $(14)$ is not meaningful in term of the significant unknown $\overline{\boldsymbol{u}_{f}}$. To overcome this difficulty, the main idea is to define a more complex application which gives multiple possibilities to the relation between $\overline{\left(\boldsymbol{u}_{f} \cdot \nabla\right) \boldsymbol{u}_{f}}$ and $\overline{\boldsymbol{u}_{f}}$. This is done by adding a hidden variable $\omega$, which encodes all the complexity of $\overline{\left(\boldsymbol{u}_{f} \cdot \nabla\right) \boldsymbol{u}_{f}}$ inside an application $\mathcal{F}$ and a space of possibilities $\Omega$ in the following way

$$
\mathcal{F}:\left\{\begin{array}{rll}
\Omega \times \mathbb{R}_{\boldsymbol{u}}^{3} & \rightarrow \mathbb{R}_{\boldsymbol{u}}^{3} \\
(\omega, u) & \mapsto \mathcal{F}(\omega, u) .
\end{array}\right.
$$

such that $\mathcal{F}\left(\omega, \overline{\boldsymbol{u}_{f}}\right)$ is close (in an unclear way) to $\overline{\left(\boldsymbol{u}_{f} \cdot \nabla\right) \boldsymbol{u}_{f}}$ and Eq. (14) could now be solved easily.

Of course, the definition of $\mathcal{F}$ strongly depends on the choice of the reduction operator - . Next, an elegant way to move forward is now to define $\Omega$ as a probability space, see [29]. Then, two main techniques emerge :

- by drawing many particular $\omega$, thus giving a random modeling of the unknown term $\overline{\left(\boldsymbol{u}_{f} \cdot \nabla\right) \boldsymbol{u}_{f}}$ through $\mathcal{F}$, compute many trajectories of the process $\overline{\boldsymbol{u}_{f}}$,

- considering the statistics or moments of the random variable $\mathcal{F}$, and solve for the evolution of the moments of the random variable $\overline{\boldsymbol{u}_{f}}$.

The advantage of the first approach is to preserve the properties of a trajectory of the process $\overline{\boldsymbol{u}_{f}}$, which is still the solution of a PDE. Thereby, the random variable $\overline{\boldsymbol{u}_{f}}$ lies in a large dimensional probability space, which requires a very large number of such succession of draws to hope for some meaningful statistics. On the contrary, solving for the evolution of the means of $\overline{\boldsymbol{u}_{f}}$ does not preserve the trajectories of the process, but it gives correct estimators and statistics on the general behavior of the gaseous velocity field.

\subsubsection{Closures}

The obtained reduced-order system as in Eq. 14 is closed by making a calculable choice on $\mathcal{F}$. Three strategies can be found in the literature for this choice, as depicted in 28, 33]:

- the functional approach: starting from the fact that the regularized version of the flow field will dissipate less energy than the real turbulent flow field does, the unresolved scales can be modeled in a first approximation by an additional diffusion process, consistently with the theory of turbulence described in paragraph 2.2 .1

$$
\overline{\left(\boldsymbol{u}_{f} \cdot \nabla\right) \boldsymbol{u}_{f}}-\left(\overline{\boldsymbol{u}_{f}} \cdot \nabla\right) \overline{\boldsymbol{u}_{f}} \approx-\nu^{\text {turb }} \nabla^{2} \overline{\boldsymbol{u}_{f}} .
$$

Here, $\nu^{\text {turb }}$ is an additional turbulent viscosity. In the case of filtering procedures, this viscosity depends on the filter size such that it vanishes for full-resolution 26,37 . As such models can depend on empirical 
constants, dynamic procedures were also proposed to get the better estimate of theses constants (see [14]).

- The structural approach: instead of simply recovering a global property of the unresolved information, structural methods aim at capturing the sub-grid scale tensor structure (see [2]).

- the "pragmatic" approach: starting from the idea that it is hard to distinguish unresolved scales effects from numerical dissipation, some authors propose to integrate effects of unresolved scales through the numerical schemes (see $[16]$ ).

\subsection{Particles in turbulence}

\subsubsection{Reduced LES models}

The fluid velocity at the location of the particle appears in the expression of the particle acceleration modeled by Stokes drag law:

$$
d \boldsymbol{C}_{i}=\frac{\boldsymbol{u}_{f}\left(t, \boldsymbol{X}_{i}\right)-\boldsymbol{C}_{i}}{\tau_{p}} d t
$$

$i \in \llbracket 1, N \rrbracket$, and $\tau_{p}$ being a characteristic relaxation time of the particule toward the underlying velocity field. When computing only a reduced description of turbulence (such as Large Eddy Simulation or LES), the exact fluid velocity field is not known. Only a regularised version of the fluid velocity is computed. Thus, the use of Stokes drag (Eq. 16) which is based on the fluid velocity at the location of the particle is not straightforward. Therefore, a closure on the fluid velocity seen by the particles is required in order to provide a consistant LES model for the disperse phase. Ideally, this model has to be in agreement with the probability space of the random variable $\mathcal{F}$ seen by the inertial particles on the fluid flow. With real turbulence, probability spaces associated with the flow are still a research topic for the community. Moreover for practical applications (such as the ones which require LES), the function $\mathcal{F}$ is often chosen in a pragmatic way such as to enforce regularity of the flow even if it implies making very strong (and sometimes questionable) hypotheses on underlying probability spaces ( 22$]$ ). Therefore, is often difficult to assert practical particle models with regard to their hypotheses in the context of real turbulence. This preliminary work aims at providing a reasonable framework, close to turbulence, consistent with the hypothesis on which practical models are designed, and with a precise probabilistic formalism, so that assessment of closure models with regards to the probability space of the random variable $\mathcal{F}$ is made possible. As a starting point, validation of the dispersion behaviour of inertial particles in this synthetic case with respect to what is expected in real turbulence and to what is assumed by standard models is studied. This is crucial, since dispersion of inertial particles in the flow is one of the simplest and most important characterizations for applications.

Up to now, very similarly to the models developed for the fluid flow, the main strategies have been to compensate second order moments of the the particle density distributions by the adjunction of energy in the form of Wiener processes (see $4,11,25,31,35,36]$ ). In its general from, this can be represented by the stochastic differential equation (17):

$$
d \boldsymbol{Z}_{t}=\mu_{t} \boldsymbol{Z}_{t} d t+\sigma_{t} d \boldsymbol{W}_{t}
$$

with $\boldsymbol{W}_{t}$ a Wiener process, $\boldsymbol{Z}_{t}$ the state vector of the particle, $\mu_{t}$ the drift and $\sigma_{t}$ the diffusion coefficient. According to the level of modeling adopted for the representation of the disperse phase $\boldsymbol{Z}_{t}$ can be chosen encompass an arbitrarily large number of variables describing the particle evolution. Traditional choices are usually limited to three variables, the first two being position and velocity ( [24]). It is to be noted that in most models, the Wiener process only acts on one variable of the particle : either its position, or its velocity, or an other intermediate variable like the velocity seen by the particle. The next section shows that in the context of equation (17), where the closure has been chosen in the form of a Wiener process, the derivation of a mesoscopic equation for the disperse phase is not a major difficulty. 


\section{Consistency of modeling APPROACHES With Numerical CASES}

Sections 1 and 2 were mainly focused on providing a meaningful formalism for reduced multiphase flow simulations in agreement with mathematical consistency and physical literature. In this context, we conclude that an appropriate formalism to describe a fluid in a large-scale reduced order in section 2.2 is the selfconditioned structure proposed by [29] and formalized Eq. (15). In a nutshell, the evolution of the large scale of the flow must be obtained as the expectation of all possible unresolved scales of the flow compatible with the resolved large scales.

Applying this formalism with the full resolution of Navier-Stokes is not easy because it is not straightforward to control large scales and unresolved scales separately. An interesting alternative that has been widely used in the literature is to rely on synthetic turbulence: by means of a summation of analytic modes, and under the constraint of specific spectral distribution and representation, one can expect to reproduce the main characteristics of the turbulence, even without verifying Navier-Stokes equations. In this section, we investigate the use of such analytic representation from $1 \mathrm{D}$ to $3 \mathrm{D}$, and we show what is the minimal representation that can be envisaged.

\subsection{Synthetic turbulence}

The synthetic flow field has been designed in order to reproduce similar dynamics as a turbulent flow field $([18,19])$. As the synthetic flow field is associated with an explicit probability space, it makes investigation of self-conditioning possible and easy. With the self-conditioned formalism, the LES flow field is seen as a stochastic process (as opposed to the traditional perception which considers it as a deterministic dynamical system), and the evolution equations associated with the LES flow field are seen on an upper-level, and describe the evolution of the whole distribution, classically driven by an infinitesimal generator.

The synthetic flow field represents turbulence with a sparse matrix of spectral modes (Eq. (18) chosen according to the energy density given by Pope's spectrum in Eq. (19) (see [28, p.232]) with Eq. 20]. Discretisation is done

$$
\begin{gathered}
\boldsymbol{u}_{f}(t, \boldsymbol{z})=\sum_{n=1}^{N} \boldsymbol{a}_{n} \cos \left(\omega_{n} t+\boldsymbol{k}_{n} \cdot \boldsymbol{z}+\varphi_{n}\right) \\
E(|\boldsymbol{k}|)=\frac{9}{4} \frac{\epsilon^{\frac{2}{3}}}{|\boldsymbol{k}|^{\frac{5}{3}}}\left(\frac{|\boldsymbol{k}| / k_{0}}{\left[\left(|\boldsymbol{k}| / k_{0}\right)^{2}+6.78\right]^{1 / 2}}\right)^{\frac{11}{3}} \exp \left(-5.2\left(\sqrt[4]{(|\boldsymbol{k}| \eta)^{4}+0.4^{4}}-0.4\right)\right) \\
\int_{0}^{|\boldsymbol{k}|_{n}} E_{\text {model }}(|\boldsymbol{k}|) d|\boldsymbol{k}|=u_{0}^{2} \frac{(2 n-1)}{2 N},
\end{gathered}
$$

with the amplitude $\boldsymbol{a}_{n}$ of the modes are chosen according to the distribution $\mathcal{N}^{\mathrm{R}}\left(0, \frac{2 u_{0}^{2}}{N}\right)$, and

$$
E_{\text {model }}(k)=\mathbb{E}\left[\sum_{n=1}^{N} \frac{1}{2} \boldsymbol{a}_{n}^{2} \boldsymbol{\delta}_{\left|\boldsymbol{k}_{n}\right|}\right]=\sum_{n=1}^{N} \frac{u_{0}^{2}}{N} \boldsymbol{\delta}_{\left|\boldsymbol{k}_{n}\right|},
$$

(for more details on Fourier representation of turbulence, see [27, Chap. 6.4]).

The direction of $\boldsymbol{a}_{n}$ is chosen at random on a sphere. The direction of $\boldsymbol{k}_{n}$ is chosen at random on a circle orthogonal to $\boldsymbol{a}_{n}$ such that the flow as a null divergence (similar to incompressibility for physical fluids). The phase is chosen according to the uniform distribution $\left|\varphi_{n}\right| \sim \mathcal{U}(0,2 \pi)$. 
Following [18], the spectral components of the energy spectrum are chosen in order to respect the numerical simulations performed in [17], which show that it seems sensible to approximate $E_{\omega}(|\boldsymbol{k}|)$ by :

$$
E_{\omega}(|\boldsymbol{k}|)=\frac{E(|\boldsymbol{k}|)}{\sqrt{2 \pi}\left(a|\boldsymbol{k}| u_{0}\right)} \exp \left(-\frac{\omega^{2}}{2\left(a|\boldsymbol{k}| u_{0}\right)^{2}}\right),
$$

with $a \in[0.4,0.51]$ depending on the wavenumber and the integral length scale (see $[17)$. The frequencies are chosen according to the distribution $\left|\omega_{n}\right| \sim \mathcal{N}\left(0,\left(a|\boldsymbol{k}| u_{0}\right)^{2}\right)$. For the numerical simulations, the random number generator chosen is ran2 presented in [30]. The numerical values are chosen such that $a=0.5$, $u_{0}=1 \mathrm{~m} . \mathrm{s}^{-1}$ and $k_{0}=1 \mathrm{~m}^{-1}$. The particle evolution is computed using Runge-Kutta scheme of order four.

The evolution of the particles on the fluid is computed by the linearized Stokes drag law in Eq. (16), with the expression of $\boldsymbol{u}_{f}$ given in Eq. 18 .

For numerical simplicity, we first start by performing one-dimensional simulations. In one dimension, a realization of the evolution of the particles submitted to a random fluid is given in Fig. 1. Although the initial positons of the particles are random and uniformly distributed on a segment, their trajectories seem very limited. They look more like oscillations around a mean drift rather than dispersion. Furthermore, when observing the evolution of the variance in a one-dimensional space for $10^{4}$ particles, see Fig. 2(a), we see that it seems bounded for this case and that it is highly dependent on the underlying fluid fluctuations.

This kind of behavior is not consistent with the properties of turbulence and the expected behavior of particles in a turbulent flow: we would rather expect a dispersion behavior similar to diffusion (see for instance [32]). Since the stochastic models of the literature have a first order effect on the second order moments of the measure of the disperse phase, it is essential to work on a numerical setup which preserves the basic properties of turbulent flows for realizations of the second order moments of the measure of the disperse phase. Hence, it is of prime importance to understand why such a behavior is observed on the simple fluid model we have chosen if we want to use it for reproducing and understanding the dynamics of inertial particles on fluids described by Navier-Stokes kind of equations.

\subsection{Simplified one-dimensional case}

As explained above, Fig. 1 enlightens an unexpected behavior in one dimensional case. Let us start by looking if it is possible to understand this behavior on a simplified case where the fluid is only represented by one sine. We have the particle evolution in Eq. (23) and the reduced evolution in Eq. (24) with the change of variables $X_{i}^{\prime}=\omega t+k X_{i}+\frac{\varphi}{2 \pi}, C_{i}^{\prime}=\omega+k C_{i}$ and $a^{\prime}=k a$.

$$
\begin{gathered}
\left\{\begin{array}{l}
d X_{i}=C_{i} d t \\
d C_{i}=\frac{a \sin \left(2 \pi\left(\omega t+k X_{i}\right)+\varphi\right)-C_{i}}{\tau_{p}}, \quad i=1, \ldots, N
\end{array}\right. \\
\left\{\begin{array}{l}
d X_{i}^{\prime}=C_{i}^{\prime} d t \\
d C_{i}^{\prime}=\frac{a^{\prime} \sin \left(2 \pi X_{i}^{\prime}\right)+\omega-C_{i}^{\prime}}{\tau_{p}},
\end{array} \quad i=1, \ldots, N .\right.
\end{gathered}
$$

We will prove the following result :

Proposition 3.1. Particles under dynamics described in Eq. (23) will follow an increasing signal, incompatible with an expected diffusive behavior.

Proof. In order to prove this result, we can first study the system (24). This system is autonomous in dimension 2, so by the Poincaré-Bendixon theorem, only three cases are possible :

- The trajectories are unbounded,

- The trajectories converge to a point,

- The trajectories converge to a limit cycle. 


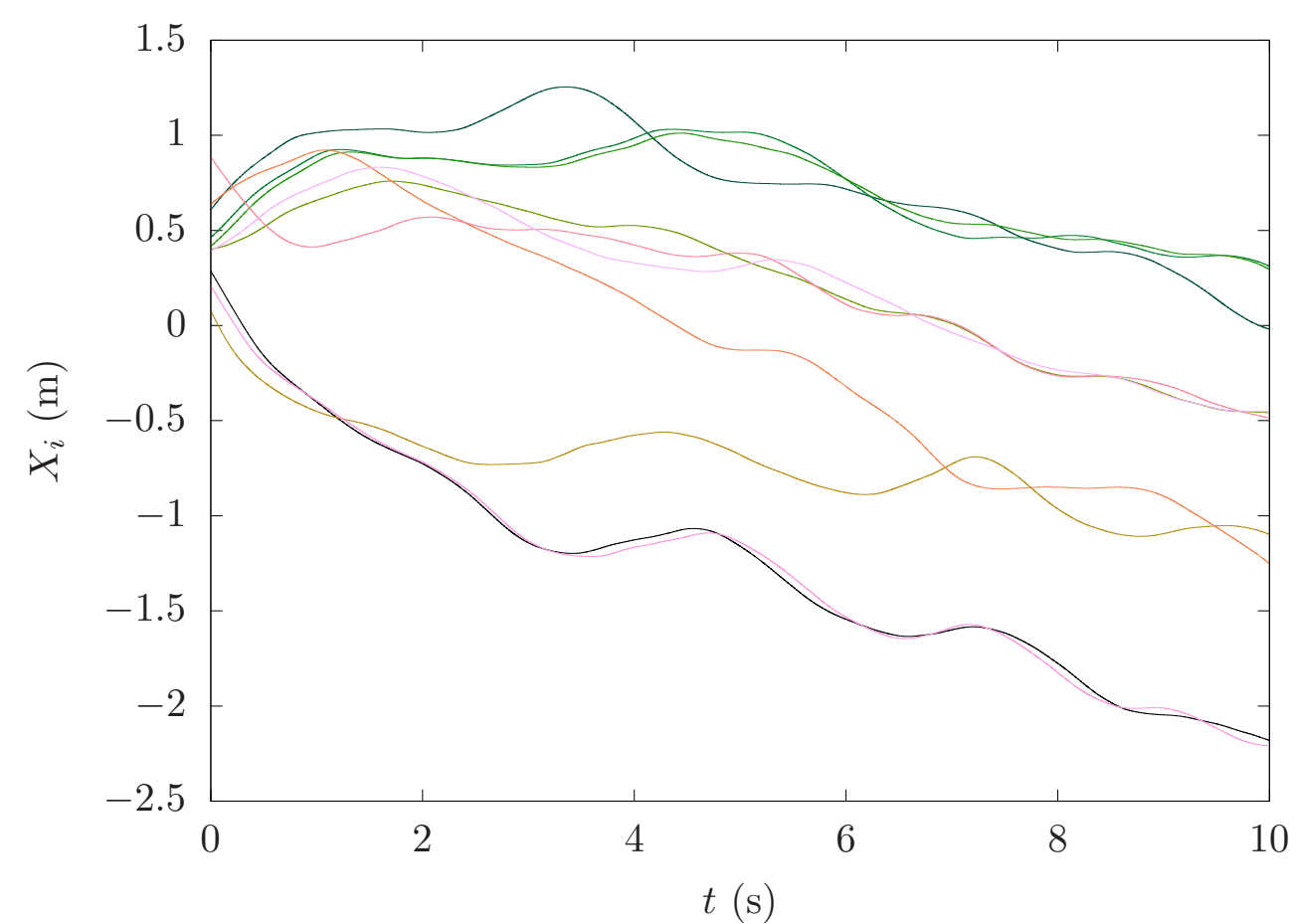

Figure 1. Position $X_{i}$ in meters (m) of particles according to time in seconds (s) and colored by their number $i \in \llbracket 0,9 \rrbracket$. Time step of $d t=0.001 \mathrm{~s}$ for particle time relaxation constant of $\tau_{p}=1 s$.

Let us now try to characterize these behaviors more precisely.

Let $i \in \llbracket 1, N \rrbracket$. Define $C_{\max }=\left|a^{\prime}\right|+|\omega|+1$. Suppose that at time $t^{*}$ we have $C_{i}^{\prime}\left(t^{*}\right)>C_{\max }$, then by continuity during a time $\delta$ we have $C_{i}^{\prime}\left(t^{*}+t\right) \geq C_{\max }$ for all $t \in[0, \delta]$. Thus

$$
\begin{aligned}
C_{\max } \leq C_{i}\left(t^{*}+\delta\right) & =C_{i}^{\prime}\left(t^{*}\right)+\int_{0}^{\delta} \frac{a^{\prime} \sin \left(2 \pi X_{i}^{\prime}\left(t^{*}+s\right)\right)+\omega-C_{i}^{\prime}\left(t^{*}+s\right)}{\tau_{p}} d s \\
& \leq C_{i}^{\prime}\left(t^{*}\right)+\int_{0}^{\delta} \frac{\left|a^{\prime}\right|+|\omega|-C_{\max }}{\tau_{p}} d s \\
& \leq C_{i}^{\prime}\left(t^{*}\right)+\delta \frac{\left|a^{\prime}\right|+|\omega|-C_{\max }}{\tau_{p}}
\end{aligned}
$$

or simply $\delta \leq\left(C_{i}^{\prime}\left(t^{*}\right)-C_{\max }\right) \tau_{p}$. It proves that in finite time, the solution falls under $C_{\max }$. Then we have proved that for all trajectories, there exists a time $t_{\max }$ where $C_{i}^{\prime}\left(t_{\max }\right)<C_{\max }$.

Denote $C_{\min }=-C_{\max }$ and use again the time $t^{*}$ with symmetric definition, we obtain

$$
\begin{aligned}
C_{\min } \geq C_{i}\left(t^{*}+\delta\right) & =C_{i}^{\prime}\left(t^{*}\right)+\int_{0}^{\delta} \frac{a^{\prime} \sin \left(2 \pi X_{i}^{\prime}\left(t^{*}+s\right)\right)+\omega-C_{i}^{\prime}\left(t^{*}+s\right)}{\tau_{p}} d s \\
& \geq C_{i}^{\prime}\left(t^{*}\right)+\int_{0}^{\delta} \frac{-\left|a^{\prime}\right|-|\omega|-C_{\min }}{\tau_{p}} d s \\
& \geq C_{i}^{\prime}\left(t^{*}\right)+\frac{\delta}{\tau_{p}}
\end{aligned}
$$




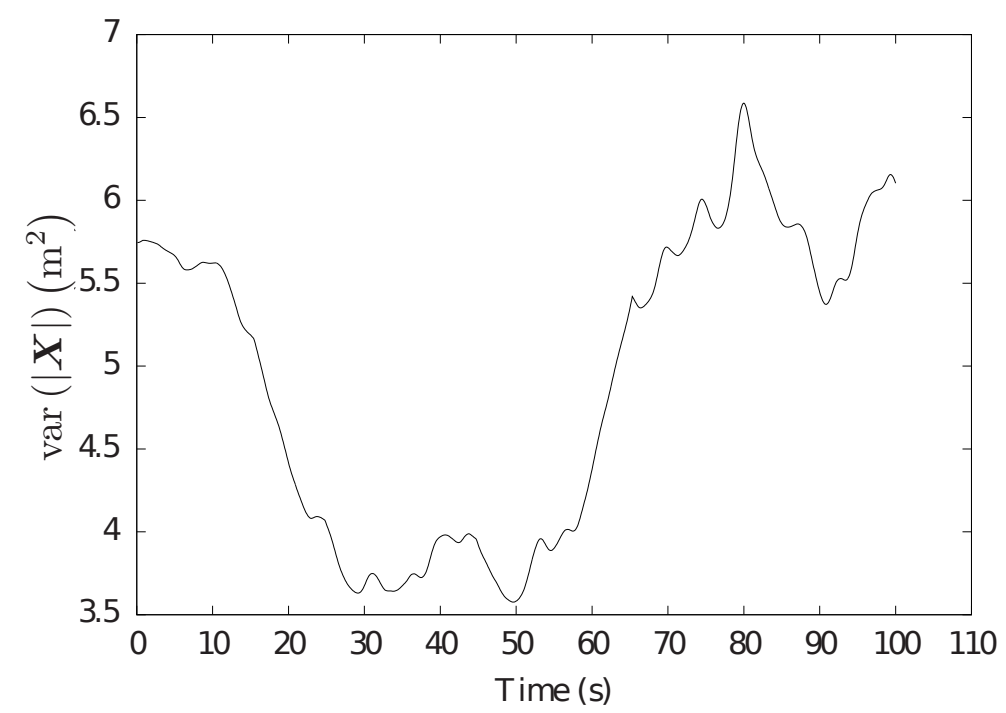

(a) $1 \mathrm{D}$

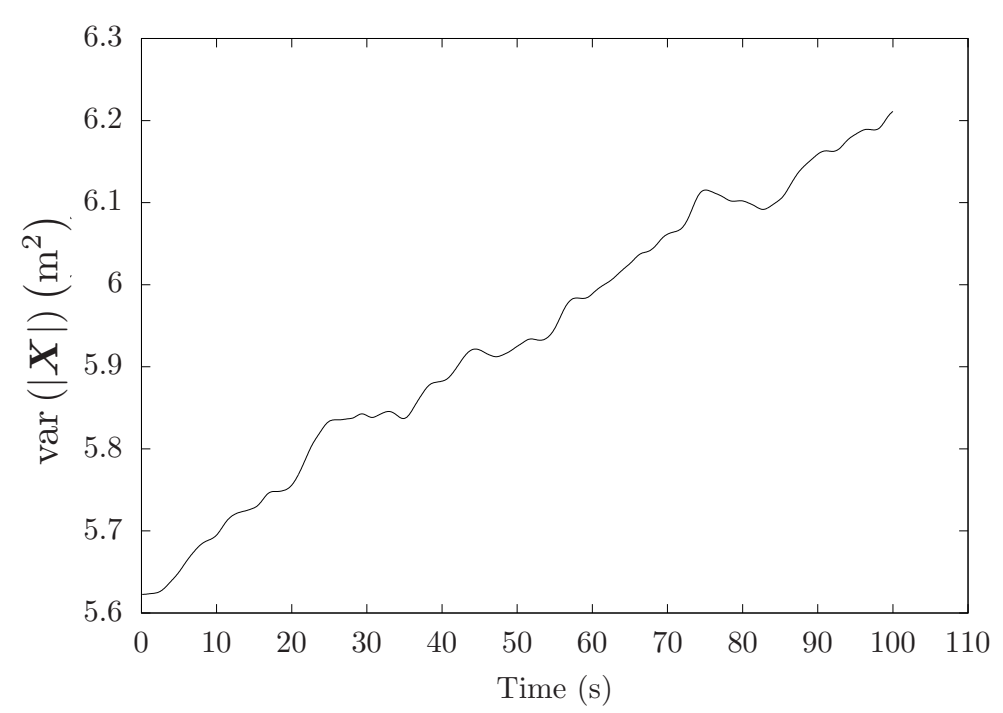

(b) $2 \mathrm{D}$

FiguRE 2. Evolution of the variance in position of $10^{4}$ particles of relaxation time constant of $\tau_{p}=1 \mathrm{~s}$ in different dimensionalities on one fluid flow realization according to time (s).

and $\delta \leq\left(C_{\min }-C_{i}^{\prime}\left(t^{*}\right)\right) \tau_{p}$. It proves that in finite time, the solution rises above $C_{\min }$. Then we have proved that for all trajectories, there exists a time $t_{\min }$ where $C_{i}^{\prime}\left(t_{\min }\right)>C_{\min }$.

Finally we can suppose that for all trajectories, the speed $C_{i}^{\prime} \in\left[-C_{\max }, C_{\max }\right]$ after some transitory time. In fact -with the same procedure- we can prove that $C_{i}^{\prime} \in\left[\omega-\left|a^{\prime}\right|, \omega+\left|a^{\prime}\right|\right]$.

Thus if $\omega>\left|a^{\prime}\right|$ then it proves that the speed stays strictly greater than $\omega-\left|a^{\prime}\right|>\varepsilon>0$, and thus the trajectories cannot be bounded. In order to prove that the particles will follow an increasing signal, we have to study the difference with this linear growing. 


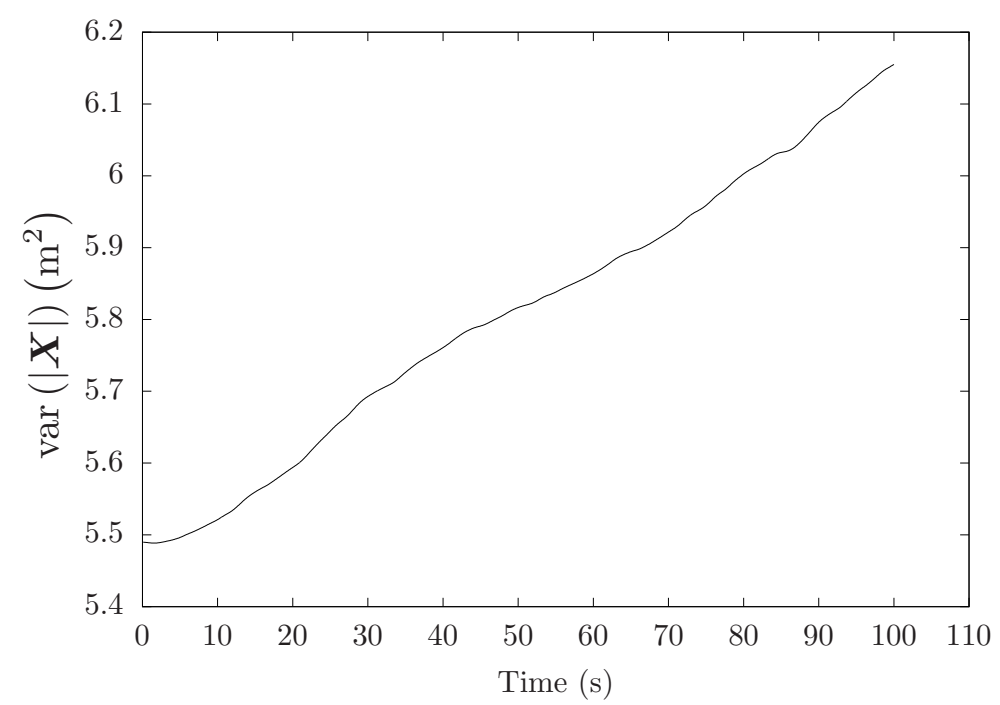

(a) $3 \mathrm{D}$

Figure 2. Evolution of the variance in position of $10^{4}$ particles of relaxation time constant of $\tau_{p}=1 \mathrm{~s}$ in different dimensionalities on one fluid flow realization according to time (s).

Denote $Y_{i}^{\prime}(t)=X_{i}^{\prime}(t)-\omega t$ then $V_{i}^{\prime}(t)=C_{i}^{\prime}(t)-\omega=\frac{d Y_{i}^{\prime}}{d t} \in\left[-\left|a^{\prime}\right|,\left|a^{\prime}\right|\right]$.

$$
\begin{aligned}
& d Y_{i}^{\prime}=\left(C_{i}^{\prime}-\omega\right) d t=V_{i}^{\prime} d t, \\
& d V_{i}^{\prime}=\frac{a^{\prime} \sin \left(2 \pi Y_{i}^{\prime}+2 \pi \omega t\right)-V_{i}^{\prime}}{\tau_{p}} d t .
\end{aligned}
$$

We can see that $V_{i}^{\prime}$ cannot converge to a constant $\bar{V}$, because there is no solution to $\bar{V}=a^{\prime} \sin (2 \pi t(\bar{V}+\omega))$ (except $\omega=\bar{V}=0$ ). Since $V_{i}^{\prime}$ cannot converge to a constant while staying in a compact, it is non-monotonous. Denote $T_{+}$a moment where $\frac{d V_{i}^{\prime}}{d t}$ changes its sign (without loss of generality, suppose it changes from $>0$ to $<0)$, i.e.

$$
a^{\prime} \sin \left(2 \pi\left(Y_{i}^{\prime}\left(T_{+}\right)+\omega T_{+}\right)\right)=V_{i}^{\prime}\left(T_{+}\right)=: V_{+} .
$$

Thus

and in particular

$$
\tau_{p} \frac{d^{2} V_{i}^{\prime}}{d t^{2}}\left(T_{+}\right)=2 \pi a^{\prime} \cos \left(2 \pi Y_{i}^{\prime}\left(T_{+}\right)+2 \pi \omega T_{+}\right)\left(V_{i}^{\prime}\left(T_{+}\right)+\omega\right)-\frac{d V_{i}^{\prime}}{d t}\left(T_{+}\right)
$$

$$
\frac{\frac{d^{2} V_{i}^{\prime}}{d t^{2}}\left(T_{+}\right)}{a^{\prime} \cos \left(2 \pi Y_{i}^{\prime}\left(T_{+}\right)+2 \pi \omega T_{+}\right)}=\frac{2 \pi}{\tau_{p}}\left(V_{+}+\omega\right)
$$

Suppose $a^{\prime}>0$ to simplify.

The quantity $\frac{d V_{i}^{\prime}}{d t}$ changes from $>0$ to $<0$, thus the second derivative is negative, so at a given time, there is a local maximum, and during a period $\left[T_{+}, T_{+}+T\right], V_{i}^{\prime}$ is decreasing and we have also $2 \pi\left(Y_{i}^{\prime}\left(T_{+}\right)+\omega T_{+}\right) \in$ $[\pi / 2,3 \pi / 2] \bmod 2 \pi$. Or simply $2 \pi\left(Y_{i}^{\prime}\left(T_{+}\right)+\omega T_{+}\right)=2 k \pi+\pi / 2+\varepsilon \pi$ with $\varepsilon \in[0,1]$.

If $V_{+}>0$ we have $\varepsilon \in[0,1 / 2]$. And since $V_{i}^{\prime}$ is decreasing, and $Y_{i}^{\prime}$ increasing unbounded, there is a moment where $2 \pi Y_{i}^{\prime}(t)+2 \pi \omega t=2 k \pi+\pi=2 \pi Y_{i}^{\prime}\left(T_{+}\right)+2 \pi \omega T_{+}+\pi / 2-\varepsilon \pi$. At this moment, $V_{i}^{\prime}$ becomes negative and 
$Y_{i}^{\prime}$ becomes decreasing. Since $V_{i}^{\prime}$ is bounded, it will reach a minimum (since it cannot converges). Denote this time $T_{-}$and we are in the symmetric case than previously.

We have proved that there exists two sequences $\left(T_{+}^{n}\right)_{n \in \mathbb{N}}$ and $\left(T_{-}^{n}\right)_{n \in \mathbb{N}}$ such that $T_{+}^{n}<T_{-}^{n}<T_{+}^{n+1}$ for all $n \in \mathbb{N}$. We can bounded the time $\left(T_{-}^{n}-T_{+}^{n}\right)$ above and below independently of $n \in \mathbb{N}$ roughly proving that the solution is close to a periodic one. Finally the solution $X_{i}^{\prime}$ is close to a increasing signal having periodic oscillation around its drift, which is incompatible with an expected diffusive behavior.

In this particular case of only one sine, we have performed a transformation which leads to an autonomous system, and hard conclusion with only a discrete set of final positions. With more exciting sines the behavior could be different. But -as it is represented in Fig. 2 a - even with more exciting sines we do not obtain in 1D a dispersive behavior as expected. Indeed, the behaviour of $\operatorname{Var}(|\boldsymbol{X}|)$ seems bounded, and does not have an increasing trend. On the contrary, it seems to oscillate back and forth around $5 \mathrm{~m}^{2}$. This is not characteristic of a dispersive behaviour where, in this case, one would expect $\operatorname{Var}(|\boldsymbol{X}|)$ to be growing with time. It makes a $1 \mathrm{D}$ model very dubious.

But, dispersion of particles is greatly influenced by the dimensionality of the underlying space chosen. Although the dynamics in the one dimensional case is very different from the physics we aim at modeling, we expect that when dimensionality is increased, this behavior will change and be most likely similar to diffusion (see Fig. 2), as envisioned by the physics, and as described by the models currently in use in the literature. Let us check this assumption in the following section.

\subsection{Higher dimensionality}

It is possible to observe numerically that by increasing the dimensionality to more than one physical dimension (Figs. 2(b) and 3(a) , the second order moment of $f_{t}$ has a better behavior, i.e. it increases quite monotonously with time, and the particles do not seem to be overly constrained by the underlying fluid flow. The higher the dimensionality, the better the dispersion of the particles. Indeed, one observes in Fig. 2(b) that the dispersion of the particles appears to be much less influenced by the characteristics of the underlying fluid flow than in the $1 \mathrm{D}$ case (see Fig. 2(a) , and that the third dimensionality brings even more smoothness (see Fig. 3(a)). The change of behavior between 2D and 3D can also be partly understood by the addition of new topologies for the three-dimensional stationary points as described in 3 .

Given these results, it seems relevant to keep on pursuing the simulation effort focusing on the three dimensional configuration.

\section{TOWARDS TWO-WAY COUPLED SYSTEMS}

The next step towards the modeling of particulate flows is to account for the impact of the disperse phase on the turbulent carrier phase, which has strong implications. Let us consider the empirical measure $\mu_{t}^{N}(\boldsymbol{Z})=$ $\frac{1}{N} \sum_{i=1}^{N} \delta_{\boldsymbol{X}_{i}(t)} \delta_{\boldsymbol{C}_{i}(t)}$ and the following evolution equation

$$
\left\{\begin{array}{rl}
d \boldsymbol{X}_{i} & =\boldsymbol{C}_{i}(t) d t \\
d \boldsymbol{C}_{i} & =\frac{\mathbf{u}_{f}\left(t, \boldsymbol{X}_{i}\right)-\boldsymbol{C}_{i}}{\tau_{p}} d t
\end{array} \quad i=1, \ldots, N .\right.
$$

In a one-way coupled context the gas phase velocity at the particle location does only depend on the particle position itself and is independent of the other particles as they share the same gas phase. In this context, we satisfy the conditions of Theorem 1.1.e. $\boldsymbol{G}\left(t, \boldsymbol{X}_{i}, \boldsymbol{C}_{i}\right)=\frac{\mathbf{u}_{f}\left(t, \boldsymbol{X}_{i}\right)-C_{i}}{\tau_{p}}$. We can thus state a theorem of convergence towards the law of the process. 
In a two-way coupled system, all particles affect the gas phase evolution such that the gas velocity is conditional to the full particle configuration. It can be parametrized by the empirical measure at time $t=0$ :

$$
\mathbf{u}_{f}(t, x)=\mathbf{u}_{f}^{N}\left(t, x, \mu_{0}^{N}[\boldsymbol{Z}]\right)
$$

In this case, the drag term now depends on all particle history, i.e. $\boldsymbol{G}^{N}\left(t, \boldsymbol{X}_{i}, \boldsymbol{C}_{i}, \mu_{0}^{N}[\boldsymbol{Z}]\right)=\frac{\mathbf{u}_{f}^{N}\left(t, \boldsymbol{X}_{i}, \mu_{t}^{0}[\boldsymbol{Z}]\right)-C_{i}}{\tau_{p}}$. Now, we are not in the context of Theorem 1.1 anymore. The open question is then to determine if it is possible to characterize a convergence of the particulate system towards a one-particle law:

$$
\begin{array}{r}
\frac{\partial}{\partial t} f_{t}+\boldsymbol{v} \cdot \nabla_{\boldsymbol{x}} f_{t}+\nabla_{\boldsymbol{v}} \cdot\left(\boldsymbol{G}^{l i m} f_{t}\right)=0 \\
\partial_{t}\left(\boldsymbol{u}_{f}\right)+\left(\boldsymbol{u}_{f} \cdot \nabla\right) \boldsymbol{u}_{f}-\nu \nabla^{2} \boldsymbol{u}_{f}=-\frac{1}{\rho_{f}} \nabla p+\int m_{p} G^{l i m} f_{t} \mathrm{~d} \boldsymbol{v}
\end{array}
$$

where $G^{l i m}$ is the forcing of the gas velocity field for an infinite number of particles, i.e. when the particulate phase behaves as a continuum, and $m_{p}$ is the mass of each particle.

\subsection{Example of the Burgers equation}

To investigate if there is an Eulerian continuum limit to the two-way problem, we set up a simplified case that considers the 1D Burgers equation on the gas velocity $u$ :

$$
\frac{\partial u_{f}}{\partial t}+\frac{\partial}{\partial x}\left(\frac{u_{f}^{2}}{2}\right)=\frac{F_{p \rightarrow f}(t, x)}{\rho_{f}}
$$

Where $F_{p \rightarrow f}$ is the force exerted by the particles on the gas phase through drag. Under the point-particle approximation, this source term is written as the sum of each particle pointwise contribution:

$$
F_{p \rightarrow f}(t, x)=\sum_{i=1}^{N} m_{p} \frac{U_{i}(t)-u_{f}(t, x)}{\tau_{p}} \delta_{X_{i}}
$$

Giving a meaning to $F_{p \rightarrow f}$ is not trivial (see [1,8,20]), and a regularization procedure is sometimes used. Here we will use the numerical cells used to discretize the Burgers equation in a finite volume sense as a regularization for the particle field, and the force applied to each cell will be the sum of the individual contribution of each particle belonging to the cell.

\subsubsection{Solution with homogeneous distribution of particles}

First we study the asymptotic limit in which the particles are perfectly uniformly distributed at time $t=0$ at the same velocity. The gas velocity also starts at a uniform velocity. In this limit the Eulerian continuum limit is valid and the particles can be represented by their Eulerian equations. We then state that the forcing term in the kinetic equation is $G^{l i m}=\frac{u_{f}-v}{\tau_{p}}$. Coupling gas phase and liquid phase equations, we get:

$$
\begin{aligned}
\frac{\partial\left(m_{p} n_{l}\right)}{\partial t}+\frac{\partial\left(m_{p} n_{l} u_{l}\right)}{\partial x} & =0 \\
\frac{\partial\left(m_{p} n_{l} u_{l}\right)}{\partial t}+\frac{\partial\left(m_{p} n_{l} u_{l}^{2}+P_{l}\right)}{\partial x} & =n_{l} m_{p} \frac{u_{f}-u_{l}}{\tau_{p}} \\
\frac{\partial u_{f}}{\partial t}+\frac{\partial}{\partial x}\left(\frac{u_{f}^{2}}{2}\right) & =\frac{m_{p} n_{l}}{\rho_{f}} \frac{u_{l}-u_{f}}{\tau_{p}}
\end{aligned}
$$


where $m_{p}$ is the (constant) mass of one particle, $n_{l}$ the number of particles per unit volume, $u_{l}$ the Eulerian velocity field and $P_{l}$ the pressure of the disperse phase. In the following we make the assumption of monokinetic disperse phase, i.e. $P_{l}=0$. The gas density $\rho_{f}$ is also assumed to be constant. Starting from an homogeneous conditions, we can easily see that the solution will still be invariant by translation at any time and the problem to be solved reduces in the following ODE:

$$
\begin{aligned}
\frac{\mathrm{d} u_{l}}{\mathrm{~d} t} & =\frac{u_{f}-u_{l}}{\tau_{p}} \\
\frac{\mathrm{d} u_{f}}{\mathrm{~d} t} & =\frac{m_{p} n_{l}}{\rho_{f}} \frac{u_{l}-u_{f}}{\tau_{p}}
\end{aligned}
$$

which solution is:

$$
\begin{aligned}
& u_{l}(t)=-\frac{1}{\kappa}\left(u_{f}^{0}-\frac{\kappa u_{l}^{0}+u_{f}^{0}}{1+\kappa}\right) e^{-\frac{1+\kappa}{\tau_{p}} t}+\frac{\kappa u_{l}^{0}+u_{f}^{0}}{1+\kappa} \\
& u_{f}(t)=\left(u^{0}-\frac{\kappa u_{l}^{0}+u_{f}^{0}}{1+\kappa}\right) e^{-\frac{1+\kappa}{\tau_{p}} t}+\frac{\kappa u_{l}^{0}+u_{f}^{0}}{1+\kappa}
\end{aligned}
$$

where $\kappa=\frac{m_{p} n_{l}}{\rho_{f}}$. The equilibrium solution is then:

$$
u_{l}(t \rightarrow \infty)=u_{f}(t \rightarrow \infty)=\frac{\kappa u_{l}^{0}+u_{f}^{0}}{1+\kappa}
$$

As a consequence, if we want to study the impact of inhomogeneity of the particulate phase by changing the number of particles but keeping the same physical problem, we need to modify the particle mass $m_{p}$ accordingly, to keep $\kappa_{m}=\int \kappa \mathrm{d} x / L_{x}=N_{p} m_{p}$ constant.

\subsection{Particle-laden case with Lagrangian particles}

Knowing the sought continuum limit of the particle system, we now investigate the impact of the number of particles, i.e. the impact of the statistical convergence of the randomly-drawn initial condition. We thus simulate the two-way coupled Burgers' problem by changing the number of particles from 1 to a large number or particles. The Burgers' equation is solved using a 1st order finite volume scheme. In Fig. 3a, we compare the time evolution of the gas velocity averaged over a large number of realizations of the initial conditions for different numbers of particles at fixed mass loading. We clearly see the convergence of the Lagrangian simulations towards the homogeneous solution, with a convergence rate of order 1 (see Fig. 3p). This convergence rate is not affected by the number of cells for numerical discretization and by the addition of physical diffusion in the Burgers equation. So even if we do not have a formal proof in the spirit of Theorem 1.1] we still have confidence in the existence of a convergence result, and thus of an Eulerian limit description.

\subsection{Eulerian modeling}

As examplified by the previous test case, the Eulerian representation is still possible for a large number of particles. If we do not have a large number of particles, let say less than one particle per cell, we still have to propose a closure for $G^{l i m}$. Moreover, having a statistically-converged NDF $f$ requires to take statistics also on 

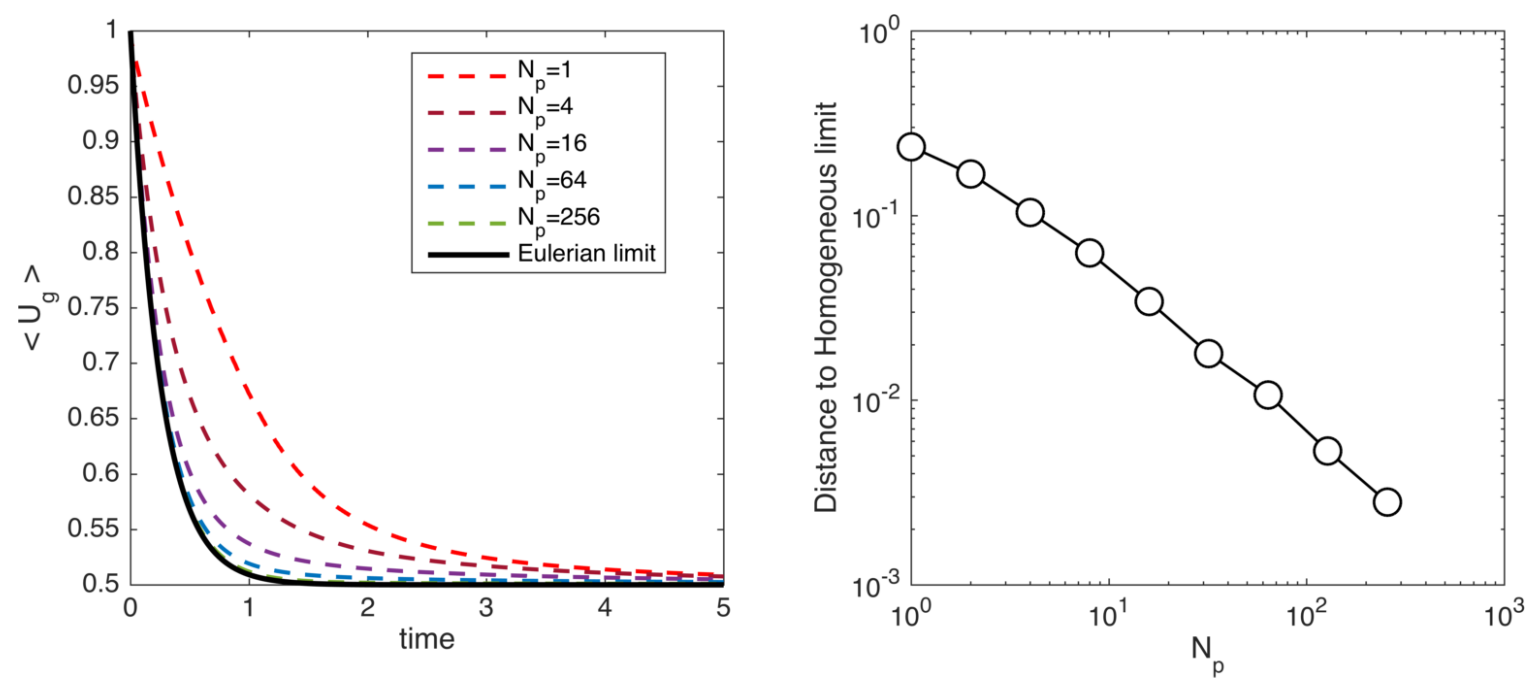

FIGURE 3. 1D burgers problem. Left: ensemble-averaged gas velocity for different number of particles from 1 to 256, to compare with the homogeneous limit (black). Right: convergence rate of the difference with the homogeneous solution with respect to the number of particles. The distance is the $L^{2}$ norm of the error.

the gas phase velocity. We thus have a two-fold closure problem:

$$
\begin{array}{r}
\frac{\partial}{\partial t} f_{t}+v \cdot \nabla_{x} f_{t}+\nabla_{v} \cdot\left(G^{l i m} f_{t}\right)=0 \\
\frac{\partial\left\langle u_{f}\right\rangle}{\partial t}+\frac{\partial}{\partial x}\left(\frac{\left\langle u_{f}\right\rangle^{2}}{2}\right)=\left\langle\frac{F_{p \rightarrow f}(t, x)}{\rho_{f}}\right\rangle-\frac{\partial}{\partial x}\left(\frac{\left\langle u_{f}^{\prime}\right\rangle^{2}}{2}\right)
\end{array}
$$

where $\langle.>$ stands for the ensemble-average over particle realizations which clearly denotes a mean over the initial law of particles, and $u_{f}^{\prime}=u_{f}-<u_{f}>$ is the fluctuation. The fluid ensemble has been generated by changing the initial position of the particles: each realization corresponds to a different draw of the initial position of each particle, using a uniform law. Thus, for each time, the mean gas velocity $\left\langle u_{g}\right\rangle$ is obtained by averaging over all individual computations for a given initial condition.

Here we clearly see that performing an Eulerian simulation sought as an ensemble-average simulation necessarily leads to an ensemble-average on the gas phase. Closing the whole system is a tough task outside of the scope of the present work.

\subsubsection{Closing the equations}

Here we give some insight of possible closures. As results in Fig. 3 clearly shows similar trends but with a different time scale, we propose to investigate the possibility to close the problem using an adapted relaxation time scale $\tau_{p}^{e f f}$ :

$$
\begin{aligned}
\frac{\mathrm{d} u_{l}}{\mathrm{~d} t} & =\frac{u_{f}-u_{l}}{\tau_{p}^{e f f}} \\
\frac{\mathrm{d} u_{f}}{\mathrm{~d} t} & =\kappa \frac{u_{l}-u_{f}}{\tau_{p}^{e f f}}
\end{aligned}
$$




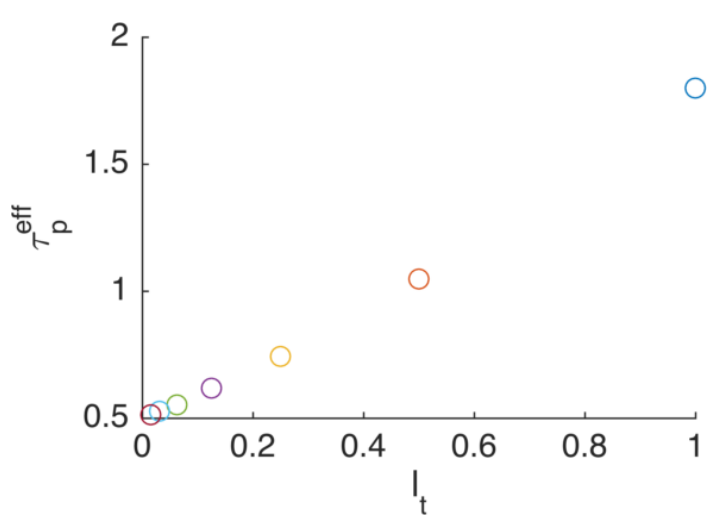

(a)

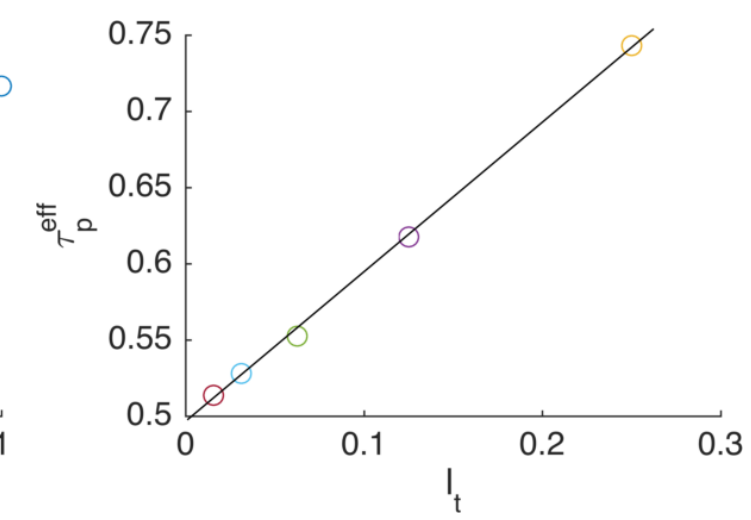

(b) zoom-in

FiguRE 4. 1D burgers problem. Evaluation of an effective relaxation time scale for the Eulerian modeling as a function of the droplet interspace.

In Fig. 4. we look at the impact of the particle interspace $l_{t}=1 / N_{p}$ on this effective time scale. We exhibit a linear trend for small $l_{t}$, which would be helpful to devise closures in a two-way coupled system. The closure for this effective time scale can then be sought as:

$$
\tau_{p}^{e f f}=\tau_{p}+\alpha l_{t}
$$

The previous example was just to show the impact of the droplet interspace on the solution, and a possible modeling strategy to account for some of the effects. We only focused on the source term, but additional fluxes can also be investigated as possible closures.

\subsubsection{Interpreting existing Eulerian simulations}

Even if we clearly show here that the ensemble average on the particle phase leads to an ensemble-average on the gas phase, i.e. RANS-like statistics, many simulations can be found in the literature in a LES context, which obviously exhibits large scale unsteady behavior. Thus, the question is: what is solved in such simulations? A possible and simple interpretation is not to consider this simulation as statistics but as a unique realization of the disperse phase represented in a Eulerian manner. This way, a unique realization of a gas phase will be considered. This turns out to be an Eulerian representation of the empirical measure, which is valid in the sense of the distributions:

$$
\frac{\partial}{\partial t} \mu_{t}^{N}+v \cdot \nabla_{x} \mu_{t}^{N}+\nabla_{v} \cdot\left(\frac{u_{g}-v}{\tau_{p}} \mu_{t}^{N}\right)=0
$$

Taking the moments of this equation and the gas equation, we get:

$$
\begin{aligned}
\frac{\partial m_{p} n_{l}^{N}}{\partial t}+\frac{\partial m_{p} n_{l}^{N} u_{l}^{N}}{\partial x} & =0 \\
\frac{\partial m_{p} n_{l}^{N} u_{l}^{N}}{\partial t}+\frac{\partial m_{p} n_{l}^{N} u_{l}^{N^{2}}+P_{l}^{N}}{\partial x} & =n_{l}^{N} m_{p} \frac{u_{f}-u_{l}^{N}}{\tau_{p}} \\
\frac{\partial u_{f}}{\partial t}+\frac{\partial}{\partial x}\left(\frac{u_{f}^{2}}{2}\right) & =\frac{m_{p} n_{l}^{N}}{\rho_{f}} \frac{u_{l}^{N}-u_{f}}{\tau_{p}}
\end{aligned}
$$

where $n_{l}^{N}$ and $u_{l}^{N}$ are zeroth and first order moments of the empirical measure, and $P_{l}^{N}$ is its pressure. This system of equations is similar to the Eulerian continuum limit, but the difference lies in the initial and boundary 


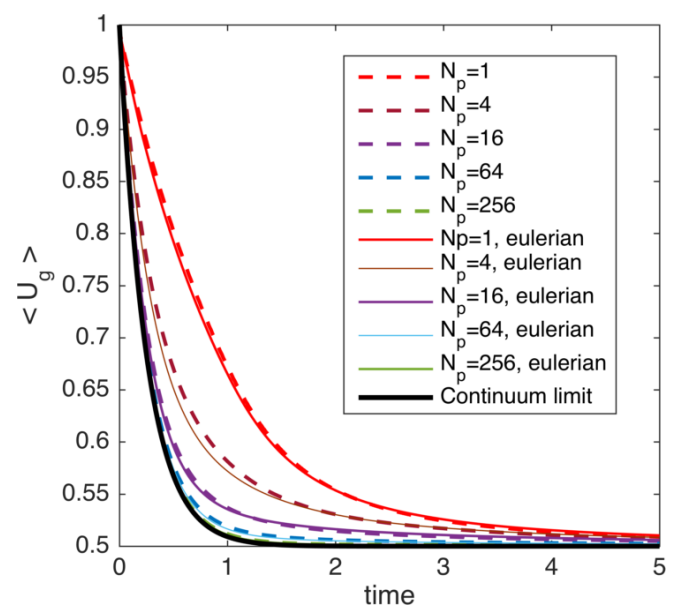

FIGURE 5. 1D burgers problem. Ensemble-averaged gas velocity for different number of particles from 1 to 256, with the Lagrangian tracking (dashed lines) and the "empirical" Eulerian moment method (full lines) to compare with the homogeneous limit (black).

conditions: while for the continuum limit, these inputs must be related to the law, here they must be randomly drawn as in the case of the Lagrangian particles.

In the case of the 1D burgers periodic problem, solving this system will not take advantage of the spatial invariance of the problem, and we thus have to solve the PDEs. In the following, we will consider a pressure-less dynamics, i.e. $P_{l}^{N}=0$, and we will use a second order scheme considering the high number density gradients to be resolved. In Fig. 5 we show the results of the gas phase statistics when using this "empirical" Eulerian moment method, demonstrating the ability of such representation to capture the right behavior.

At this point, it is worth to mention that existing LES two-way coupled simulations do not consider a random sampling of the initial/boundary conditions. Instead, they use statistically-converged inputs, leading to an incoherent modeling. It is possible to consider them as regularized simulations in the sense that the initial/boundary conditions has been smeared out enough to lose any random effect.

\section{Conclusions}

In this paper, an exploratory research activity has been started with the aim of statistical and probabilistic modeling of a cloud of particles coupled with a turbulent fluid. Regarding the complexity of this problem, the wide range of expertise of the authors represents an important asset. Here we have set up a common basis to address the issues arising from the context of this work. By investigating all the passing to the limit, we have clarified the main milestones to reach in order to answer our problematic. We have also defined a proper numerical framework to evaluate the modeling approaches and to investigate the statistical properties of our systems of interest. Finally, we have shown the main limitations in two-way coupled system, proposing some possible solutions to overcome them.

Acknowledgement : The support of the work of D. Mercier through a DGA PhD grant and the financial support of Initiative HPC@Maths (Ecole Polytechnique - PIs F. Alouges and M. Massot) are gratefully acknowledged.

\section{REFERENCES}

[1] N. Aguillon. Riemann problem for a particle-fluid coupling. Mathematical Models and Methods in Applied Sciences, 25(01):39$78,2015$. 
[2] J. Bardina, J. H. Ferziger, and W. C. Reynolds. Improved subgrid scale models for Large Eddy Simulation. In 13th Fluid and Plasma Dynamics Conference, 1980. AIAA Paper No. 80-1357.

[3] J. Bec, A. Celani, M. Cencini, and S. Musacchio. Clustering and collisions of heavy particles in random smooth flows. Physics of Fluids, 17(7):073301, 2005.

[4] M. Bini and W. Jones. Particle acceleration in turbulent flows: A class of nonlinear stochastic models for intermittency. Physics of Fluids, 19(3):035104, 2007.

[5] F. Bolley. Limite de champ moyen de systèmes de particules. Séminaire Équations aux dérivées partielles (Polytechnique), 2009:1-15, 2010.

[6] S. Chapman and T. G. Cowling. The mathematical theory of nonuniform gases. Cambridge Mathematical Library. Cambridge University Press, Cambridge, third edition, 1990. An account of the kinetic theory of viscosity, thermal conduction and diffusion in gases, In co-operation with D. Burnett, With a foreword by Carlo Cercignani.

[7] F. Doisneau. Eulerian modeling and simulation of polydisperse moderately dense coalescing spray flows with nanometric-toinertial droplets : application to Solid Rocket Motors. PhD thesis, Ecole Centrale Paris, 2013.

[8] K. Domelevo and M.-H. Vignal. Limites visqueuses pour des systèmes de type fokker-planck-burgers unidimensionnels. Comptes Rendus de l'Académie des Sciences-Series I-Mathematics, 332(9):863-868, 2001.

[9] W. E. Stochastic PDEs in turbulence theory. In Proc. 1st Intl. Congress Chinese Math., volume 20, pages 27-46, Beijing, 1998. Amer. Math. Soc.,Providence, RI (2001).

[10] O. Emre, R. Fox, M. Massot, S. de Chaisemartin, S. Jay, and F. Laurent. Eulerian modeling of a polydisperse evaporating spray under realistic internal-combustion-engine conditions. Flow, Turbulence and Combustion, 93(4):689-722, 2014.

[11] P. Fede and O. Simonin. Numerical study of the subgrid fluid turbulence effects on the statistics of heavy colliding particles. Physics of Fluids, 18(4):045103, 2006.

[12] J. Ferziger and H. Kaper. Mathematical Theory of Transport Processes in Gases. North-Holland Publishing Company, 1972.

[13] R. Fox. On multiphase turbulence models for collisional fluid-particle flows. Journal of Fluid Mechanics, 742:368-424, 2014.

[14] M. Germano, U. Piomelli, P. Moin, and W. H. Cabot. A dynamic subgrid-scale eddy viscosity model. Physics of Fluids A: Fluid Dynamics, 3(7):1760-1765, 1991.

[15] M. Gorokhovski and R. Zamansky. Lagrangian simulation of large and small inertial particles in a high reynolds number flow: Stochastic simulation of subgrid turbulence/particle interactions. In Center for Turbulence Research, Proceedings of the Summer Program, pages 37-46, Stanford University, 2014.

[16] F. F. Grinstein and C. Fureby. Recent progress on MILES for high Reynolds number flows. J. of Fluids Eng., 124(2):848-861, 2002.

[17] J. Hunt, J. Buell, and A. Wray. Big whorls carry little whorls. Center for Turbulence Research, Proceedings of the Summer Program, 1987.

[18] R. Ijzermans, E. Meneguz, and M. Reeks. Segregation of particles in incompressible random flows: singularities, intermittency and random uncorrelated motion. Journal of Fluid Mechanics, 653:99-136, 2010.

[19] R. Kraichnan. Diffusion by a random velocity field. The physics of fluids, 13(1):22-31, 1970.

[20] F. Lagoutière, N. Seguin, and T. Takahashi. A simple 1D model of inviscid fluid-solid interaction. Journal of Differential Equations, 245(11):3503-3544, 2008.

[21] O. E. Lanford. Time evolution of large classical systems, pages 1-111. Springer Berlin Heidelberg, Berlin, Heidelberg, 1975.

[22] W. D. McComb. The physics of fluid turbulence. Chemical physics, 1990.

[23] J.-P. Minier. Statistical descriptions of polydisperse turbulent two-phase flows. Physics Reports, 665(Supplement C):1 - 122, 2016. Statistical descriptions of polydisperse turbulent two-phase flows.

[24] J.-P. Minier and E. Peirano. The pdf approach to turbulent polydispersed two-phase flows. Physics reports, 352(1):1-214, 2001.

[25] J.-P. Minier, E. Peirano, and S. Chibbaro. Pdf model based on langevin equation for polydispersed two-phase flows applied to a bluff-body gas-solid flow. Physics of fluids, 16(7):2419-2431, 2004.

[26] F. Nicoud and F. Ducros. Subgrid-scale stress modelling based on the square of the velocity gradient. Flow Turbulence and Combustion, 62(3):183-200, 1999.

[27] S. Pope. Turbulent flows. Cambridge University, 2000.

[28] S. Pope. Turbulent flows. Cambridge University Press, 2001.

[29] S. Pope. Self-conditioned fields for large-eddy simulations of turbulent flows. Journal of Fluid Mechanics, 652:139-169, 2010.

[30] W. Press, B. Flannery, S. Teukolsky, and W. Vetterling. Numerical recipes, volume 3. cambridge University Press, cambridge, 1989.

[31] M. Reeks. On the dispersion of small particles suspended in an isotropic turbulent fluid. Journal of fluid mechanics, 83(3):529546, 1977.

[32] L. Richardson. Atmospheric diffusion shown on a distance-neighbour graph. Proceedings of the Royal Society of London A: Mathematical, Physical and Engineering Sciences, 110(756):709-737, 1926.

[33] P. Sagaut. Large eddy simulation for incompressible flows: an introduction. Springer Science \& Business Media, 2006.

[34] J. Serrin. Mathematical principles of classical fluid mechanics. In C. Truesdell, editor, Fluid Dynamics I / Strömungsmechanik I, pages 125-263. Springer Berlin Heidelberg, Berlin, Heidelberg, 1959. 
[35] B. Shotorban and S. Balachandar. A eulerian model for large-eddy simulation of concentration of particles with small stokes numbers. Physics of Fluids, 19(11):118107, 2007.

[36] B. Shotorban and F. Mashayek. A stochastic model for particle motion in large-eddy simulation. Journal of Turbulence, 7(18), 2006.

[37] J. Smagorinsky. General circulation experiments with the primitive equations: I. the basic experiment. Monthly weather review, 91(3):99-164, 1963

[38] A.-S. Sznitman. Topics in propagation of chaos. In P.-L. Hennequin, editor, Ecole d'Eté de Probabilités de Saint-Flour XIX — 1989, pages 165-251, Berlin, Heidelberg, 1991. Springer Berlin Heidelberg.

[39] C. Truesdell. Rational Thermodynamics. Springer-Verlag New York, 2nd edition, 1984.

[40] C. Villani. Limite de champ moyen. In Cours de DEA, 2001-2002. ENS Lyon, 2002.

[41] C. Villani. A review of mathematical topics in collisional kinetic theory. In S. Friedlander and D. Serre, editors, Handbook of Mathematical Fluid Dynamics, Vol. 1. Elsevier, 2002.

[42] L. Zaichik, O. Simonin, and V. Alipchenkov. An eulerian approach for large eddy simulation of particle transport in turbulent flows. Journal of Turbulence, 10(4):1-21, 2009.

[43] R. Zamansky, F. Coletti, . Massot, and A. Mani. Turbulent thermal convection driven by heated inertial particles. Journal of Fluid Mechanics, 809:390-437, 2016 